\title{
Apollonian Circle Packings: Geometry and Group Theory III. Higher Dimensions*
}

Ronald L. Graham, ${ }^{1}$ Jeffrey C. Lagarias, ${ }^{2}$ Colin L. Mallows, ${ }^{3}$

Allan R. Wilks, ${ }^{4}$ and Catherine H. Yan ${ }^{5}$

${ }^{1}$ Department of Computer Science and Engineering,

University of California at San Diego,

La Jolla, CA 92110, USA

graham@ucsd.edu

${ }^{2}$ Department of Mathematics, University of Michigan, Ann Arbor, MI 48109-1109, USA

lagarias@umich.edu

${ }^{3}$ Avaya Labs, Basking Ridge, NJ 07920, USA

colinm@research.avayalabs.com

${ }^{4}$ AT\&T Labs, Florham Park, NJ 07932-0971, USA

allan@ research.att.com

${ }^{5}$ Department of Mathematics, Texas A\&M University, College Station, TX 77843, USA

cyan@math.tamu.edu

\begin{abstract}
This paper gives $n$-dimensional analogues of the Apollonian circle packings in Parts I and II. Those papers considered circle packings described in terms of their Descartes configurations, which are sets of four mutually touching circles. They studied packings that had integrality properties in terms of the curvatures and centers of the circles. Here we consider collections of $n$-dimensional Descartes configurations, which consist of $n+2$ mutually touching spheres.

We work in the space $\mathcal{M}_{\mathbb{D}}^{n}$ of all $n$-dimensional oriented Descartes configurations parametrized in a coordinate system, augmented curvature-center coordinates, as those $(n+2) \times(n+2)$ real matrices $\mathbf{W}$ with $\mathbf{W}^{T} \mathbf{Q}_{D, n} \mathbf{W}=\mathbf{Q}_{W, n}$ where $Q_{D, n}=x_{1}^{2}+$ $\cdots+x_{n+2}^{2}-(1 / n)\left(x_{1}+\cdots+x_{n+2}\right)^{2}$ is the $n$-dimensional Descartes quadratic form, $Q_{W, n}=-8 x_{1} x_{2}+2 x_{3}^{2}+\cdots+2 x_{n+2}^{2}$, and $\mathbf{Q}_{D, n}$ and $\mathbf{Q}_{W, n}$ are their corresponding symmetric matrices. On the parameter space $\mathcal{M}_{\mathbb{D}}^{n}$ of augmented curvature-center matrices, the group $\operatorname{Aut}\left(Q_{D, n}\right)$ acts on the left and $\operatorname{Aut}\left(Q_{W, n}\right)$ acts on the right. Both these groups are isomorphic to the $(n+2)$-dimensional Lorentz group $O(n+1,1)$, and give two different "geometric" actions. The right action of $\operatorname{Aut}\left(Q_{W, n}\right)$ (essentially) corresponds to Möbius transformations
\end{abstract}

\footnotetext{
* Ronald L. Graham was partially supported by NSF Grant CCR-0310991. Catherine H. Yan was partially supported by NSF Grants DMS-0070574, DMS-0245526 and a Sloan Fellowship. She is also affiliated with Nankai University, China.
} 
acting on the underlying Euclidean space $\mathbb{R}^{n}$ while the left action of $\operatorname{Aut}\left(Q_{D, n}\right)$ is defined only on the parameter space $\mathcal{M}_{\mathbb{D}}^{n}$.

We introduce $n$-dimensional analogues of the Apollonian group, the dual Apollonian group and the super-Apollonian group. These are finitely generated groups in $\operatorname{Aut}\left(Q_{D, n}\right)$, with the following integrality properties: the dual Apollonian group consists of integral matrices in all dimensions, while the other two consist of rational matrices, with denominators having prime divisors drawn from a finite set $S$ depending on the dimension. We show that the Apollonian group and the dual Apollonian group are finitely presented, and are Coxeter groups. We define an Apollonian cluster ensemble to be any orbit under the Apollonian group, with similar notions for the other two groups. We determine in which dimensions there exist rational Apollonian cluster ensembles (all curvatures are rational) and strongly rational Apollonian sphere ensembles (all augmented curvature-center coordinates are rational).

\section{Introduction}

In Part I we considered Apollonian circle packings, and observed that there exist such packings where the circles all had integer curvatures and the quantities (curvature) $\times$ (circle center) had integer entries. We gave an explanation for this phenomenon, in terms of the Descartes configurations in the packing. A Descartes configuration is a configuration of four mutually tangent circles, with all six tangency points distinct. We introduced the space $\mathcal{M}_{\mathbb{D}}$ of all ordered, oriented Descartes configurations, parametrized in a coordinate system expressed in terms of the curvatures and centers of the circles in the packing.

The explanation of the integrality properties was as follows. This configuration space of ordered, oriented Descartes configurations was shown to be a principal homogeneous space for the Lorentz group $O(3,1)$, a six-dimensional real Lie group which we identified with the real automorphism group $\operatorname{Aut}\left(Q_{D}\right)$ of a quaternary quadratic form

$$
Q_{D}\left(x_{1}, x_{2}, x_{3}, x_{4}\right)=x_{1}^{2}+x_{2}^{2}+x_{3}^{2}+x_{4}^{2}-\frac{1}{2}\left(x_{1}+x_{2}+x_{3}+x_{4}\right)^{2},
$$

which we called the Descartes form. We showed that the set of all Descartes configurations in the packing formed an orbit of a discrete subgroup $\mathcal{A}$ acting on this space, which was independent of the packing, and which we termed the Apollonian group. This group consisted of $4 \times 4$ integer matrices, and the integrality properties of the Apollonian group explained the occurrence of packings with integral curvatures and (curvature) $\times$ (circle center) data. If a single Descartes configuration in the packing has such integrality properties, then every Descartes configuration in the packing inherits such properties, and thus all the circles in the packing have such properties.

Part I also introduced two other discrete subgroups of $\operatorname{Aut}\left(Q_{D}\right)$, which had integrality properties and a geometric interpretation. These were the dual Apollonian group $\mathcal{A}^{\perp}$ and the group generated by both the Apollonian group and the dual Apollonian group together, which we called the super-Apollonian group $\mathcal{A}^{S}$.

In this paper we study to what extent these results carry over to the $n$-dimensional case. We show that there exist $n$-dimensional analogues of the Apollonian group, dual Apollonian group and super-Apollonian group. We study properties of their orbits on the parameter space $\mathcal{M}_{\mathbb{D}}^{n}$ of $n$-dimensional ordered, oriented Descartes configurations which we call Apollonian cluster ensembles. We determine to what extent properties of 
the two-dimensional case extend to the $n$-dimensional case. One immediate difference is that in dimensions $n \geq 4$ the orbits of the Apollonian group no longer correspond to sphere packings; the spheres in these configurations overlap. However, the groups involved still have rationality properties, and we consider the question when there exist ensembles having all curvatures rational and/or having all data (curvature) $\times($ sphere center) rational.

In Section 2 we describe six properties that hold in the two-dimensional case, and then summarize what the main results of this paper say about generalizations of these to higher dimensions. Some of them generalize completely, others only in an infinite set of specific dimensions, and some are specific to dimension 2.

In Section 3 we describe the parameter space $\mathcal{M}_{\mathbb{D}}^{n}$ of all $n$-dimensional ordered, oriented Descartes configurations in a coordinate system, augmented curvature-center matrices, as those $(n+2) \times(n+2)$ real matrices $\mathbf{W}$ with $\mathbf{W}^{T} \mathbf{Q}_{D, n} \mathbf{W}=Q_{W, n}$ where $Q_{D, n}=x_{1}^{2}+\cdots+x_{n+2}^{2}-(1 / n)\left(x_{1}+\cdots+x_{n+2}\right)^{2}$ is the (matrix of the) $n$-dimensional Descartes quadratic form and $Q_{W, n}=-8 x_{1} x_{2}+2 x_{3}^{2}+\cdots+2 x_{n+2}^{2}$. On the space $\mathcal{M}_{\mathbb{D}}^{n}$ of augmented curvature-center matrices, the group $\operatorname{Aut}\left(Q_{D, n}\right)$ acts on the left and $\operatorname{Aut}\left(Q_{W, n}\right)$ acts on the right. Both these groups are isomorphic to the $(n+2)$-dimensional Lorentz group $O(n+1,1)$, and give two different "geometric" actions. The right action of $\operatorname{Aut}\left(Q_{W, n}\right)$ (essentially) corresponds to Möbius transformations acting on the underlying Euclidean space $\mathbb{R}^{n}$ while the left action of $\operatorname{Aut}\left(Q_{D, n}\right)$ is defined only on the parameter space.

In Section 4 we define the $n$-dimensional analogues of the Apollonian group, the dual Apollonian group and the super-Apollonian group introduced in Part I. As we just noted above, one immediate difference with the two-dimensional case is that in dimensions $n \geq$ 4 the orbits of the Apollonian group no longer correspond to sphere packings; the spheres in these configurations overlap. Furthermore, even viewed in the parameter space $\mathcal{M}_{\mathbb{D}}^{n}$ the action of the $n$-dimensional Apollonian group $\mathcal{A}_{n}$ is not discrete. However, the action of the dual Apollonian group $\mathcal{A}_{n}^{\perp}$ is discrete on $\mathcal{M}_{\mathbb{D}}^{n}$ for all $n \geq 2$. To restore a discrete group action for the Apollonian group and the super-Apollonian group in certain higher dimensions, it suffices to view it as a diagonal action on $\mathcal{M}_{\mathbb{D}}^{n}(\mathbb{R}) \times \prod_{p \mid n-1} \mathcal{M}_{\mathbb{D}}^{n}\left(\mathbb{Q}_{p}\right)$, in which $\mathcal{M}_{\mathbb{D}}^{n}\left(\mathbb{Q}_{p}\right)$ is a $p$-adic parameter space. This can be done in dimensions for which there are rational points in the parameter space $\mathcal{M}_{\mathbb{D}}^{n}=\mathcal{M}_{\mathbb{D}}^{n}(\mathbb{R})$.

In Section 5 we determine presentations for the Apollonian group $\mathcal{A}_{n}$ in all dimensions $n \geq 3$. In dimension 3 there are extra relations of the form $\left(\mathbf{S}_{i} \mathbf{S}_{j}\right)^{3}=\mathbf{I}$ whenever $i \neq j$. The group $\mathcal{A}_{n}$ is a hyperbolic Coxeter group in all dimensions $n \geq 3$.

In Section 6 we investigate rationality properties of Apollonian cluster ensembles. We prove that strongly rational configurations exist if and only if the dimension $n=2 k^{2}$ or $n=(2 k+1)^{2}$.

In Section 7 we consider to what extent the duality operator $\mathbf{D}$ defined in dimension 2 has an $n$-dimensional analogue. In dimension 2 this operator takes a Descartes configuration $\mathcal{D}$ to a dual Descartes configuration $\mathcal{D}^{\prime}$ consisting of four circles, each of which is orthogonal to all but one of the circles in the packing. We observe that this operation has a geometric analogue in dimension $n \geq 3$, which maps the configuration $\mathcal{D}$ to the set $\mathcal{E}$ of $n+2$ spheres having the property of being orthogonal to all but one of the spheres in $\mathcal{D}$. These spheres in $\mathcal{E}$ do not form a Descartes configuration, but have an equi-inclination property instead. In dimensions $n \geq 3$ this operation does not have an algebraic interpretation in $\operatorname{Aut}\left(Q_{D, n}\right)$. 
In Section 8 we make some concluding remarks, stating some unresolved questions.

In the Appendix we describe the action of the $n$-dimensional Möbius group Möb $(n)$ on (ordered, oriented) Descartes configurations, encoded via an isomorphism, as the action of $\operatorname{Aut}\left(Q_{W, n}\right)$.

The general framework of this paper was developed by the second author (JCL), who also did much of the writing. This paper is a revised and extended version of a preprint originally written in 2000 .

\section{Main Results}

The object of this paper is to study to what extent the properties of Apollonian circle packings studied in Parts I and II have $n$-dimensional analogues. The two-dimensional case had the following six features:

(P1) [Parameter Space Property] The space $\mathcal{M}_{\mathbb{D}}$ of all ordered, oriented Descartes configurations can be identified with the set of real intertwining matrices $\mathbf{W}$ under congruence between two rational quadratic forms in four variables, the Descartes form $Q_{D}$ and a certain form $Q_{W}$, i.e. $\mathbf{W}^{T} \mathbf{Q}_{D} \mathbf{W}=\mathbf{Q}_{W}$. We called the $4 \times 4$ matrix $\mathbf{W}$ the augmented curvature-center coordinates of the associated ordered, oriented Descartes configuration. The parameter space $\mathcal{M}_{\mathbb{D}}$ is a real algebraic variety and a principal homogeneous space for the Lorentz group $O(3,1)$.

(P2) [Orbit Property] There exist three groups of matrices in $\operatorname{Aut}\left(Q_{D}\right)$ of determinants \pm 1 , the Apollonian group $\mathcal{A}$, the dual Apollonian group $\mathcal{A}^{\perp}$ and the super-Apollonian group $\mathcal{A}^{S}$, which have geometrically characterizable actions on Descartes configurations. In particular, the set of Descartes configurations in an Apollonian circle packing comprise a single orbit of a single Descartes configuration under the action of the Apollonian group (disregarding orientation and ordering).

(P3) [Integer Matrix Property] The Apollonian group, dual Apollonian group and super-Apollonian group each consist of integer matrices. Thus all three groups are discrete subgroups of $G L(4, \mathbb{R})$. They each have a discontinuous action on the parameter space $\mathcal{M}_{\mathbb{D}}$ of ordered, oriented Descartes configurations.

(P4) [Coxeter Group Property] Each of the Apollonian group, dual Apollonian group and super-Apollonian group have group presentations as hyperbolic Coxeter groups.

(P5) [Integral Packing Property] There exist integer Apollonian circle packings, ones in which all Descartes configurations have integer curvatures. Furthermore, there exist super-integral Descartes configurations, ones whose augmented curvaturecenter coordinate matrices $\mathbf{W}$ are integer matrices. There are super-integral Apollonian packings, ones in which every Descartes configuration has this property. The set of all super-integral ordered, oriented Descartes configurations forms 672 orbits under the action of the super-Apollonian group.

(P6) [Duality Operator] There exists a duality operator $\mathbf{D}$ in $\operatorname{Aut}\left(Q_{\mathcal{D}}\right)$ given by a fixed matrix $G L(4, \mathbb{R})$ with half-integer entries. It acts by conjugacy on the 
super-Apollonian group and gives an outer automorphism of order 2 of this group. This outer automorphism conjugates the Apollonian group to the dual Apollonian group. The duality operator has a geometric interpretation in terms of its action on individual Descartes configurations.

The results of this paper generalize each of these features, which we consider in order. The parameter space property (P1) generalizes to all dimensions, as was shown by three of the authors in [23]. Results in this direction were established earlier by Wilker [35], who used the term cluster for an ordered, but not oriented, Descartes configuration. There is a notion of augmented curvature-center coordinates for an ordered, oriented $n$-dimensional Descartes configuration, and a parameter space $\mathcal{M}_{\mathbb{D}}^{n}$ of all such configurations, specified by a matrix condition that intertwines two quadratic forms in $n+2$ variables under conjugacy, as was shown in [23]. Furthermore, the space $\mathcal{M}_{\mathbb{D}}^{n}$ has the structure of a principal homogeneous space for $O(n+1,1)$. We present these results in Section 3.

The orbit property (P2) generalizes to all dimensions. There exists an $n$-dimensional version of the Apollonian group, the dual Apollonian group and the super-Apollonian group, with the same geometric interpretation of their actions on Descartes configurations. These are described in Section 4. We define an Apollonian cluster ensemble to be an orbit of a single (ordered, oriented) Descartes configuration under the action of the Apollonian group. However, there is a sphere packing interpretation of the geometric packing corresponding to this orbit in dimension 3 only, and not in dimensions $n \geq 4$.

The integer matrix property ( $\mathrm{P} 3$ ) partially generalizes to all dimensions, as we discuss in Section 4. The $n$-dimensional Apollonian group, dual Apollonian group and superApollonian group each consist of integral matrices in dimension 3, and they act discretely. The dual Apollonian group consists of integer matrices in all dimensions, and so is a discrete subgroup of $G L(n+2, \mathbb{R})$ and acts discontinuously on the parameter space $\mathcal{M}_{\mathbb{D}}^{n}$. However, for $n \geq 4$ the Apollonian group and super-Apollonian group consist of rational matrices, with denominators whose prime factors all divide $n-1$. The Apollonian group $\mathcal{A}_{n}$ is not a discrete subgroup of $G L(n+1, \mathbb{R})$ for $n \geq 4$ and does not have a discontinuous action on $\mathcal{M}_{\mathbb{D}}^{n}$ (Theorem 4.1). However, we can restore discreteness by adding an action on some $p$-adic groups, for those primes $p$ dividing $n-1$. That is, the super-Apollonian group $\mathcal{A}_{n}^{S}$ can be embedded discretely by a diagonal action inside $G L(n+2, \mathbb{R}) \times \prod_{p \mid n-1} G L\left(n+2, \mathbb{Q}_{p}\right)$ for all $n$. For certain dimensions $n$, those characterized in Section 6 as $n=2 k^{2}$ or $n=(2 k+1)^{2}$ for $k \geq 1$, one can get a discontinuous diagonal action of the super-Apollonian group on a parameter space $\mathcal{M}_{\mathbb{D}}^{n}(\mathbb{R}) \times \prod_{p \mid n-1} \mathcal{M}_{\mathbb{D}}^{n}\left(\mathbb{Q}_{p}\right)$. For this it is necessary that the p-adic parameter spaces $\mathcal{M}_{\mathbb{D}}^{n}\left(\mathbb{Q}_{p}\right)$ have enough points. Finally on the level of "packings" in the weak sense of spheres not crossing each other, this holds in dimensions 2 and 3 for all these groups, and holds for the dual Apollonian group $\mathcal{A}_{n}^{\perp}$ for all $n \geq 4$ (Theorem 4.3), but not for the other two groups.

The hyperbolic Coxeter group property (P4) partially (and perhaps completely) generalizes to all dimensions. We show in Section 5 that for all $n \geq 3$ the $n$-dimensional Apollonian group is a hyperbolic Coxeter group (Theorems 5.1 and 5.2). For $n=3$ the group has an extra relation, which explains the existence of the structures "The Bowl of Integers" and "The Hexlet" studied by Soddy [31]-[33] and Gosset [17]. It may be true 
that in all dimensions the dual Apollonian group and super-Apollonian group are also hyperbolic Coxeter groups, but we leave these as open questions.

The integral packing properties in (P5) generalize to all dimensions in weakened forms. In Section 6 we observe that in all dimensions $n \geq 3$ there exist Apollonian cluster ensembles in which every sphere has a rational curvature, with denominators divisible only by a certain finite set of primes, those dividing $n-1$ (Theorem 6.1). We then consider the question in which dimensions $n$ does there exist a super-rational Descartes configuration, i.e. one whose augmented curvature-center coordinate matrix $\mathbf{W}_{\mathcal{D}}$ is a rational matrix in $\mathcal{M}_{\mathbb{D}}^{n}$ ? We prove this can be done if and only if the dimension $n=2 k^{2}$ or $n=(2 k+1)^{2}$ for some $k \geq 1$ (Theorem 6.3).

The duality operation in (P6) giving a conjugacy between the Apollonian group and the dual Apollonian group, appears to exist algebraically only in dimension 2. In Section 7 we show that viewed as a geometric action, there does exist a natural "duality operator" acting on Descartes configurations in all dimensions $n \geq 2$. However, in dimensions $n \geq 3$, the image of this operator is not a Descartes configuration, but instead is a collection of $n+2$ spheres, each of which intersects the others in a particular fixed angle $\theta_{n}$, which depends on the dimension $n$ (Theorem 7.2).

\section{Descartes Configurations and Group Actions in $\mathbb{R}^{n}$}

\subsection{Descartes Configurations and Augmented Curvature-Center Coordinates}

We start with the generalization of the Descartes circle theorem to the $n$-dimensional case. A Descartes configuration in $\mathbb{R}^{n}$ consists of $(n+2)$ pairwise tangent $(n-1)$-spheres $\left(S_{1}, S_{2}, \ldots, S_{n+2}\right)$ in $\mathbb{R}^{n}$, with all points of tangency distinct. This result, which was termed the Soddy-Gossett theorem in [23], after Soddy [30] and Gossett [16] (although it was discovered earlier in the three-dimensional case), states that if the spheres have disjoint interiors then

$$
\sum_{j=1}^{n+2} \frac{1}{r_{i}^{2}}=\frac{1}{n}\left(\sum_{i=1}^{n+2} \frac{1}{r_{i}}\right)^{2} .
$$

The Descartes circle theorem is the case $n=2$.

The Soddy-Gossett theorem holds for all Descartes configurations, including configurations where one sphere encloses the others, provided that we assign appropriate signs to the curvatures, so that the configuration has a (total) orientation, as defined below. An oriented sphere is a sphere together with an assigned direction of unit normal vector, which can point inward or outward. If it has radius $r$ then its oriented radius is $r$ for an inward pointing normal and $-r$ for an outward pointing normal. Its oriented curvature (or "signed curvature") is $1 / r$ for an inward pointing normal and $-1 / r$ for an outward pointing normal. By convention, the interior of an oriented sphere is its interior for an inward pointing normal and its exterior for an outward pointing normal. An oriented Descartes configuration is a Descartes configuration in which the orientations of the spheres are compatible in the following sense: either (i) the interiors of all $n+2$ oriented spheres are disjoint, or (ii) the interiors are disjoint when all orientations are reversed. 
Each Descartes configuration has exactly two compatible orientations in this sense, one obtained from the other by reversing all orientations. The positive (total) orientation of a Descartes configuration is the one in which the sum of the signed curvatures is positive, while the negative (total) orientation is the one in which the sum of the curvatures is negative. One can check that the sum of the curvatures cannot be zero.

Now let $b_{i}=1 / r_{i}$ denote the (signed) curvature of the $i$ th sphere of an ordered (totally) oriented Descartes configuration, and let $\mathbf{b}=\left(b_{1}, \ldots, b_{n+2}\right)$. The geometry of such a Descartes configuration is encoded in the signed curvature vector $\mathbf{b}$. In the positively oriented case, where $\sum_{j=1}^{n+2} b_{j}>0$, one of the following holds: (i) all of $b_{1}, b_{2}, \ldots, b_{n+2}$ are positive; (ii) $n+1$ are positive and one is negative; (iii) $n+1$ are positive and one is zero; or (iv) $n$ are positive and equal and the other two are zero. These four cases correspond respectively to the following configurations of mutually tangent spheres: (i) $n+1$ spheres, with another in the curvilinear simplex that they enclose; (ii) $n+1$ spheres inscribed inside another larger sphere; (iii) $n+1$ spheres with one hyperplane (the $(n+2)$ nd "sphere"), tangent to each of them; and (iv) $n$ equal spheres with two common parallel tangent planes.

We can reformulate the Soddy-Gossett theorem in matrix terms. Let $b_{i}=1 / r_{i}$ denote the (signed) curvature of the $i$ th sphere of an ordered, oriented Descartes configuration, and let $\mathbf{b}=\left(b_{1}, \ldots, b_{n+2}\right)$, then (3.1) becomes

$$
\mathbf{b}^{T} \mathbf{Q}_{D, n} \mathbf{b}=0,
$$

in which $\mathbf{Q}_{D, n}$ is the symmetric matrix associated to the $n$-dimensional Descartes quadratic form, defined below.

Definition 3.1. The $n$-dimensional Descartes quadratic form $Q_{D, n}$ is the quadratic form in $n+2$ variables whose associated symmetric matrix is

$$
\mathbf{Q}_{D, n}:=\mathbf{I}_{n+2}-\frac{1}{n} \mathbf{1}_{n+2} \mathbf{1}_{n+2}^{T},
$$

in which $\mathbf{1}=(1,1, \ldots, 1)^{T}$ is a column vector of length $n+2$.

Here the original Descartes quadratic form is

$$
\mathbf{Q}_{D, 2}=I-\frac{1}{2} \mathbf{1 1}^{T}=\frac{1}{2}\left[\begin{array}{rrrr}
1 & -1 & -1 & -1 \\
-1 & 1 & -1 & -1 \\
-1 & -1 & 1 & -1 \\
-1 & -1 & -1 & 1
\end{array}\right] .
$$

In [23] three of the authors of this paper showed that there exists a parametrization of the set $\mathcal{M}_{\mathbb{D}}^{n}$ of all ordered, oriented Descartes configurations in $\mathbb{R}^{n}$, using a coordinate system involving the curvatures and centers of the spheres, which appears as Theorem 3.1 below.

Definition 3.2. Given an oriented sphere $S$ in $\mathbb{R}^{n}$, its curvature-center coordinates consist of the $(n+1)$-vector

$$
\mathbf{m}(S)=\left(b, b x_{1}, \ldots, b x_{n}\right),
$$


in which $b$ is the signed curvature of $S$ (assumed nonzero) and $\mathbf{x}(S)=\mathbf{x}=\left(x_{1}, x_{2}, \ldots, x_{n}\right)$ is its center. For the degenerate case of an oriented hyperplane $H$, its curvature-center coordinates $\mathbf{m}(H)$ are defined to be

$$
\mathbf{m}(S)=(0, \mathbf{h}),
$$

where $\mathbf{h}:=\left(h_{1}, h_{2}, \ldots, h_{n}\right)$ is the unit normal vector that gives the orientation of the hyperplane.

To see the origin of this definition in the degenerate case, let the point of $H$ closest to the origin be $\mathbf{z}=\lambda \mathbf{h}$ for some real value $\lambda$. For $t>|\lambda|$, let $S_{t}$ be the oriented sphere of radius $t$ centered at $(t+\lambda) \mathbf{h}$, which has center in direction $\mathbf{h}$ from the origin and contains z. As $t \rightarrow \infty$ the oriented spheres $S_{t}$ clearly converge geometrically to the oriented hyperplane $H$, and $\mathbf{m}\left(S_{t}\right)=(1 / t,(1+\lambda / t) \mathbf{h}) \rightarrow \mathbf{m}(H)=(0, \mathbf{h})$.

Curvature-center coordinates are not quite a global coordinate system, because they do not always uniquely specify an oriented sphere. Given $\mathbf{m} \in \mathbb{R}^{n+1}$, if its first coordinate $b$ is nonzero then there exists a unique sphere having $\mathbf{m}=\mathbf{m}(S)$. However, if $b=0$, the hyperplane case, there is a hyperplane if and only if $\sum h_{i}^{2}=1$, and in that case there is a pencil of hyperplanes that have the given value $\mathbf{m}$, which differ from each other by a translation.

We obtain a global coordinate system for spheres by adding an additional coordinate. This coordinate incorporates information about the sphere $\bar{S}$ obtained from $S$ by inversion in the unit sphere. In $n$-dimensional Euclidean space, the operation of inversion in the unit sphere replaces the point $\mathbf{x}$ by $\mathbf{x} /|\mathbf{x}|^{2}$, where $|\mathbf{x}|^{2}=\sum_{j=1}^{n} x_{j}^{2}$. Consider a general oriented sphere $S$ with center $\mathbf{x}$ and oriented radius $r$. Then inversion in the unit sphere takes $S$ to the sphere $\bar{S}$ with center $\overline{\mathbf{x}}=\mathbf{x} /\left(|\mathbf{x}|^{2}-r^{2}\right)$ and signed radius $\bar{r}=r /\left(|\mathbf{x}|^{2}-r^{2}\right)$. If $|\mathbf{x}|^{2}>r^{2}$, then $\bar{S}$ has the same sign as $S$. In all cases,

$$
\frac{\mathbf{x}}{r}=\frac{\overline{\mathbf{x}}}{\bar{r}}
$$

and

$$
\bar{b}=\frac{|\mathbf{x}|^{2}}{r}-r .
$$

Definition 3.3. Given an oriented sphere $S$ in $\mathbb{R}^{n}$, its augmented curvature-center coordinates of $S$ are given by the $(n+2)$-vector

$$
\mathbf{w}(S):=\left(\bar{b}, b, b x_{1}, \ldots, b x_{n}\right)=(\bar{b}, \mathbf{m}),
$$

in which $\bar{b}=b(\bar{S})$ is the curvature of the sphere or hyperplane $\bar{S}$ obtained by inversion of $S$ in the unit sphere, and the entries of $\mathbf{m}$ are its curvature-center coordinates. For hyperplanes we define

$$
\mathbf{w}(H):=\left(\bar{b}, 0, h_{1}, \ldots, h_{n}\right)=(\bar{b}, \mathbf{m}),
$$

where $\bar{b}$ is the oriented curvature of the sphere or hyperplane $\bar{H}$ obtained by inversion of $H$ in the unit sphere. 
Augmented curvature-center coordinates provide a global coordinate system for oriented spheres: no two distinct oriented spheres have the same coordinates. The only case to resolve is when $S$ is a hyperplane, i.e. $b=0$. Relation (3.7) shows that $\left(\bar{b}, b x_{1}, \ldots, b x_{n}\right)$ are the curvature-center coordinates of $\bar{S}$, and if $\bar{b} \neq 0$, this uniquely determines $\bar{S}$; inversion in the unit circle then determines $S$. In the remaining case, $b=\bar{b}=0$ and $S=\bar{S}$ is the unique hyperplane passing through the origin whose unit normal is given by the remaining coordinates.

Given a collection $\left(S_{1}, S_{2}, \ldots, S_{n+2}\right)$ of $n+2$ oriented spheres (possibly hyperplanes) in $\mathbb{R}^{n}$, the augmented matrix $\mathbf{W}$ associated with it is the $(n+2) \times(n+2)$ matrix whose $j$ th row has entries given by the augmented curvature-center coordinates $\mathbf{w}\left(S_{j}\right)$ of the $j$ th sphere.

To state the next result, we introduce another quadratic form.

Definition 3.4. The (n-dimensional) Wilker quadratic form $Q_{W, n}$ is the $(n+2)$-variable quadratic form given by the symmetric $(n+2) \times(n+2)$ matrix

$$
\mathbf{Q}_{W, n}:=\left[\begin{array}{rrc}
0 & -4 & 0 \\
-4 & 0 & 0 \\
0 & 0 & 2 \mathbf{I}_{n}
\end{array}\right] .
$$

This name is made in honor of J. B. Wilker [35], who introduced in spherical geometry a coordinate system analogous to augmented curvature-center coordinates, see Sections 2, pp. 388-390, and 9 of [35]. However, he did not formulate any result explicitly exhibiting a quadratic form like $\mathbf{Q}_{W, n}$; see the remark on p. 349 of [23].

Theorem 3.1 (Augmented Euclidean Descartes Theorem). The augmented matrix $\mathbf{W}=\mathbf{W}_{\mathcal{D}}$ of an oriented Descartes configuration $\mathcal{D}$ of $n+2$ spheres $\left\{S_{i}: 1 \leq i \leq n+2\right\}$ in $\mathbb{R}^{n}$ satisfies

$$
\mathbf{W}^{T} \mathbf{Q}_{D, n} \mathbf{W}=\left[\begin{array}{rrr}
0 & -4 & 0 \\
-4 & 0 & 0 \\
0 & 0 & 2 \mathbf{I}_{n}
\end{array}\right]
$$

Conversely, any real solution $\mathbf{W}$ to (3.12) is the augmented matrix $\mathbf{W}_{\mathcal{D}}$ of a unique ordered, oriented Descartes configuration $\mathcal{D}$.

Proof. This is proved as Theorem 3.3 in [23].

Theorem 3.1 states that the augmented curvature-center coordinates of an ordered, oriented Descartes configuration give an intertwining map between the Descartes form and the Wilker form. We note that the Soddy-Gossett theorem is a special case of Theorem 3.1, encoded as the (2,2) entry of the matrix $\mathbf{W}^{T} \mathbf{Q}_{D, n} \mathbf{W}$.

Both the Descartes quadratic form and the Wilker quadratic form are equivalent over the real numbers to the Lorentzian quadratic form

$$
Q_{\mathcal{L}, n}(x):=-x_{0}^{2}+x_{1}^{2}+\cdots+x_{n+1}^{2},
$$


see Section 3.2. This quadratic form has a large group of real automorphisms under congruence

$$
\operatorname{Aut}\left(Q_{\mathcal{L}, n}\right)=\left\{\mathbf{U} \in G L(n, \mathbb{R}): \mathbf{U}^{T} \mathbf{Q}_{\mathcal{L}, n} \mathbf{U}=\mathbf{Q}_{\mathcal{L}, n}\right\},
$$

which is the Lorentz group $O(n+1,1)$. In consequence both the Descartes quadratic form $Q_{D, n}$ and Wilker quadratic form $Q_{W, n}$ have automorphism groups under (real) congruence which are conjugates of the Lorentz group.

The Descartes form is not only equivalent to the Lorentz form over the real numbers, but sometimes over the rational numbers. In dimension 2 the Descartes and Lorentz forms are rationally equivalent, where one has

$$
\mathbf{Q}_{\mathcal{L}, 2}(x)=\mathbf{J}_{0}^{T} \mathbf{Q}_{D, 2} \mathbf{J}_{0},
$$

with

$$
\mathbf{J}_{0}=\frac{1}{2}\left[\begin{array}{rrrr}
1 & 1 & 1 & 1 \\
1 & 1 & -1 & -1 \\
1 & -1 & 1 & -1 \\
1 & -1 & -1 & 1
\end{array}\right] .
$$

In dimension 2 the Wilker form $Q_{W, 2}$ is also rationally equivalent to both the Descartes form and the Lorentz form, as shown in Section 3.1 of Part I.

In higher dimensions these three forms are not always rationally equivalent. A necessary condition for rational equivalence of two quadratic forms is that their determinants differ by a rational square. We have $\operatorname{det}\left(\mathbf{Q}_{\mathcal{L}}\right)=-1, \operatorname{det}\left(\mathbf{Q}_{D}\right)=-2 / n$ and $\operatorname{det}\left(\mathbf{Q}_{W}\right)=-2^{n+4}$. It follows that a necessary condition for rational equivalence of the Descartes and Lorentz forms in dimension $n$ is that $n=2 k^{2}$; for the Wilker and Lorentz forms in dimension $n$ that $n=2 k$; and for the Descartes and Wilker forms in dimension $n$ that $n=k^{2}$ for odd $k$ or $n=2 k^{2}$ for even $k$. All three of these necessary conditions hold if and only if $n=2 k^{2}$.

In Section 6 we show that the last condition is sufficient for equivalence of the Descartes and Wilker forms.

\subsection{Möbius and Lorentz Group Actions}

The Augmented Euclidean Descartes Theorem yields two group actions on the space of Descartes configurations. The group $\operatorname{Aut}\left(Q_{D, n}\right)$ acts on the left and the group $\operatorname{Aut}\left(Q_{W, n}\right)$ acts on the right, as

$$
\mathbf{W}_{\mathcal{D}} \mapsto \mathbf{U W}_{\mathcal{D}} \mathbf{V}^{-1}, \quad \text { with } \quad \mathbf{U} \in \operatorname{Aut}\left(Q_{D, n}\right), \quad \mathbf{V} \in \operatorname{Aut}\left(Q_{W, n}\right)
$$

The two group actions obviously commute with each other. The following result generalizes the two-dimensional case, where the Lorentzian form $Q_{\mathcal{L}, 2}$ is given in (3.14).

\section{Theorem 3.2.}

(1) The groups $\operatorname{Aut}\left(Q_{D, n}\right)$ and $\operatorname{Aut}\left(Q_{W, n}\right)$ are conjugate over the real numbers to $\operatorname{Aut}\left(Q_{\mathcal{L}, n}\right) \equiv O(n+1,1)$. 
(2) The group $\operatorname{Aut}\left(Q_{D, n}\right)$ acts transitively on the left on the parameter space $\mathcal{M}_{\mathbb{D}}^{n}$ of all ordered, oriented Descartes configurations. Given two such Descartes configurations $\mathcal{D}$ and $\mathcal{D}^{\prime}$ there exists a unique $\mathbf{U} \in \operatorname{Aut}\left(Q_{D, n}\right)$ such that $\mathbf{U W}_{\mathcal{D}}=\mathbf{W}_{\mathcal{D}^{\prime}}$.

(3) The group $\operatorname{Aut}\left(Q_{W}\right)$ acts transitively on the right on the space of all ordered, oriented Descartes configurations $\mathcal{M}_{\mathbb{D}}^{n}$. Given two such Descartes configurations $\mathcal{D}$ and $\mathcal{D}^{\prime}$ there exists a unique $\mathbf{V} \in \operatorname{Aut}\left(Q_{W, n}\right)$ such that $\mathbf{W}_{\mathcal{D}} \mathbf{V}^{-1}=\mathbf{W}_{\mathcal{D}^{\prime}}$.

Remark. This result allows one to define both a left and right $O(n+1,1)$ action on the space $\mathcal{M}_{\mathbb{D}}^{n}$, which depends on the choice of conjugacy made in (1). Then (2) and (3) show both these actions are transitive and have a trivial stabilizer. This gives $\mathcal{M}_{\mathbb{D}}^{n}$ the structure of a principal homogeoneous space (or torsor) for $O(n+1,1)$, for each of these actions.

Proof. Part (1) follows for $Q_{W, n}$ on taking $\mathbf{Q}_{\mathcal{L}, n}=\mathbf{Z}^{T} \mathbf{Q}_{W, n} \mathbf{Z}$ with

$$
\mathbf{Z}=\frac{1}{\sqrt{2}}\left[\begin{array}{rrr}
\frac{1}{2} & -\frac{1}{2} & 0 \\
\frac{1}{2} & \frac{1}{2} & 0 \\
0 & 0 & \mathbf{I}_{n}
\end{array}\right]
$$

It then follows for $Q_{D, n}$ because it is conjugate to $Q_{W, n}$ by Theorem 3.1, using $\mathbf{W}_{\mathcal{D}}$ for any fixed Descartes configuration.

Parts (2) and (3) follow immediately from (1), because $\operatorname{Aut}\left(Q_{\mathcal{L}, n}\right)=O(n+1,1)$ acts transitively.

Since the spheres in a Descartes configuration appear as the rows in the augmented matrix $\mathbf{W}_{\mathcal{D}}$ of an oriented Descartes configuration, the action on the right by elements of $\operatorname{Aut}\left(Q_{W, n}\right)$ maps spheres to spheres. This action can essentially be identified with the Möbius group of linear fractional transformations acting on the one-point compactification $\hat{\mathbb{R}}^{n}$ of $\mathbb{R}^{n}$. More precisely, it corresponds to a direct product of the Möbius group with $\{\mathbf{I},-\mathbf{I}\}$, because the Möbius group preserves total orientation of Descartes configurations. A precise description of the isomorphism is given in the Appendix. (The case $n=2$ was treated in Appendix A of Part I.)

The action on the left, by $\operatorname{Aut}\left(Q_{D, n}\right)$, mixes together the different spheres in the original Descartes configuration, and does not make sense as an action on individual spheres. This group action is intrinsically associated to the $(n(n+1) / 2)$-dimensional (real) parameter space $\mathcal{M}_{\mathbb{D}}^{n}$ of oriented Descartes configurations.

\section{Apollonian Groups and Apollonian Cluster Ensembles}

In Parts I and II we studied Apollonian circle packings in terms of the Descartes configurations they contain. We showed they consisted of a single orbit of a discrete subgroup of the automorphism group $\operatorname{Aut}\left(Q_{D, 2}\right)$ of the Descartes quadratic form $Q_{D, 2}$. We also introduced another discrete subgroup of $\operatorname{Aut}\left(Q_{D, 2}\right)$, the dual Apollonian group, and, in addition, the super-Apollonian group, which is the subgroup of $\operatorname{Aut}\left(Q_{D, 2}\right)$ generated by the Apollonian group and the dual Apollonian group together. 
We show there are analogues of these three groups in all dimensions $n \geq 3$, which are subgroups of $\operatorname{Aut}\left(Q_{D, n}\right)$ consisting of rational matrices. We call these the $n$-dimensional Apollonian group, dual Apollonian group and super-Apollonian group. We then define an Apollonian cluster ensemble to be an orbit of the Apollonian group. This provides a generalization of Apollonian packing to all dimensions, though it turns out not to correspond to a sphere-packing in dimensions $n \geq 4$.

\section{1. n-Dimensional Apollonian Group}

The $n$-dimensional Apollonian group $\mathcal{A}_{n}=\left\langle\mathbf{S}_{1}, \mathbf{S}_{2}, \ldots, \mathbf{S}_{n+2}\right\rangle$ consists of $(n+2) \times$ $(n+2)$ matrices with

$$
\mathbf{S}_{j}:=\mathbf{I}+\frac{2}{n-1} \mathbf{e}_{j} \mathbf{1}^{T}-\frac{2 n}{n-1} \mathbf{e}_{j} \mathbf{e}_{j}^{T},
$$

where $\mathbf{e}^{j}$ is the $j$ th unit coordinate (column) vector and $\mathbf{1}=\mathbf{e}_{1}+\cdots+\mathbf{e}_{n+2}=$ $(1,1, \ldots, 1)^{T}$. That is, $\mathbf{S}_{j}$ is the identity matrix in all rows but the $j$ th row, and where has -1 on the diagonal, and all off diagonal elements equal to $2 /(n-1)$. It is straightforward to check that $\mathcal{A}_{n} \subset \operatorname{Aut}\left(Q_{D, n}\right)$. The relations $\mathbf{S}_{j}^{2}=\mathbf{I}$ are evident.

The algebraic action of the operator $\mathbf{S}_{j}$ on the augmented curvature-center coordinates $\mathbf{W}_{\mathcal{D}}$ of a Descartes configuration $\mathcal{D}$ is to take it to $\mathbf{S}_{j} \mathbf{W}_{\mathcal{D}}$, which is $\mathbf{W}_{\mathcal{D}^{\prime}}$ for some $\mathcal{D}^{\prime}$. The geometric interpretation of this action is to fix all (oriented) spheres in the Descartes configuration $\mathcal{D}$ except the $j$ th sphere, and to replace that sphere with the unique other sphere that is tangent to the remaining $n+1$ spheres, assigned an appropriate orientation; this is $\mathcal{D}^{\prime}$. For a fixed Descartes configuration $\mathcal{D}$ this operation can also be realized by a Möbius transformation that is an inversion with respect to the sphere that passes through the $n(n+1) / 2$ tangency points of the remaining $n+1$ spheres in the Descartes configuration. (The existence of such a sphere is demonstrated in Theorem 7.1.)

It is apparent that $\mathcal{A}_{n}$ is a group of integer matrices for $n=2$ and 3 , while for $n \geq 4$ it is not always integral, consisting of rational matrices whose denominators contain only powers of primes that divide $n-1$.

Theorem 4.1. The Apollonian group $\mathcal{A}_{n}$ is a discrete subgroup of $G L(n+2, \mathbb{R})$ for $n=2$ and $n=3$. It is not a discrete subgroup of $G L(n+2, \mathbb{R})$ for all $n \geq 4$.

Proof. The group $\mathcal{A}_{n}$ is discrete in dimensions 2 and 3 since it is then a subgroup of $G L(n+1, \mathbb{Z})$.

We consider the element $\mathbf{S}_{1} \mathbf{S}_{2}$, and show for $n \geq 4$ that the $\operatorname{set}\left\{\left(\mathbf{S}_{1} \mathbf{S}_{2}\right)^{k}: k \geq 1\right.$ does not contain the identity matrix, but the closure of this set does contain the identity matrix. The $(n+1) \times(n+1)$ matrix $\mathbf{S}_{1} \mathbf{S}_{2}$ is a product of two reflections, so it has determinant 1 and has $n$ of its eigenvalues equal to 1 . Its first two rows are $\left(a_{n}^{2}-1,-a_{n}, a_{n}^{2}+a_{n}, a_{n}^{2}+a_{n}, \ldots\right)$ and $\left(a_{n},-1, a_{n}, a_{n}, \ldots\right)$ in which $a_{n}=2 /(n-1)$, and all other rows are those of the identity matrix. Its two nonunit eigenvalues are $e^{ \pm i \theta_{n}}$ with $\cos \left(\theta_{n} / 2\right)=a_{n} / 2$, so $\theta_{n}=2 \cos ^{-1}(1 /(n-1))$. Since these two eigenvalues are distinct, it follows that $\mathbf{S}_{1} \mathbf{S}_{2}$ is diagonalizable. Now $\Sigma_{n}:=\left\{\left(\mathbf{S}_{1} \mathbf{S}_{2}\right)^{k}: k \geq 1\right\}$ contains diagonalizable elements with all eigenvalues arbitrarily close to 1 , so it contains the identity matrix as a limit point. 
When $n \geq 4$ it is well known that $\theta_{n}$ is an irrational multiple of $\pi$, whence $\Sigma_{n}$ does not contain the identity matrix, which shows that the Apollonian group $\mathcal{A}_{n}$ is not a discrete subgroup of $G L(n+1, \mathbb{R})$.

Because the Apollonian group is arithmetic one can view it as a discrete group if one considers its actions on certain $p$-adic groups $G L\left(n+2, \mathbb{Q}_{p}\right)$ for $p \mid n-1$. Let $n^{*}=n-1$ if $n$ is even, and $(n-1) / 2$ if $n$ is odd. Then one can establish that the Apollonian group $\mathcal{A}_{n}$ is a discrete group when embedded diagonally in $G L(n+2, \mathbb{R}) \times \prod_{p \mid n^{*}} G L\left(n+2, \mathbb{Q}_{p}\right)$. Using this structure, one may obtain a discrete (i.e. discontinuous) diagonal action of the Apollonian group on the space $\mathcal{M}_{\mathbb{D}}^{n}(\mathbb{R}) \times \prod_{p \mid n^{*}} \mathcal{M}_{\mathbb{D}}^{n}\left(\mathbb{Q}_{p}\right)$, whenever all of the parameter spaces $\mathcal{M}_{\mathbb{D}}^{n}\left(\mathbb{Q}_{p}\right)$ are nonempty. Here the spaces $\mathcal{M}_{\mathbb{D}}^{n}\left(\mathbb{Q}_{p}\right)$ are defined as the $p$-adic solutions to the conditions in (3.12). The criterion in Section 6.2, shows that $\mathcal{M}_{\mathbb{D}}^{n}(\mathbb{R})$ contains rational solutions, hence $p$-adic solutions for all $p$, when $n=2 k^{2}$ or $(2 k+1)^{2}$, for some $k \geq 1$.

\section{2. n-Dimensional Dual Apollonian Group}

The $n$-dimensional dual Apollonian group $\mathcal{A}_{n}^{\perp}=\left\langle\mathbf{S}_{1}^{\perp}, \mathbf{S}_{2}^{\perp}, \ldots, \mathbf{S}_{n+2}^{\perp}\right\rangle$ consists of $(n+$ 2) $\times(n+2)$ matrices with

$$
\mathbf{S}_{j}^{\perp}:=\mathbf{I}+2 \mathbf{1} \mathbf{e}_{j}^{T}-4 \mathbf{e}_{j} \mathbf{e}_{j}^{T} .
$$

That is, $\mathbf{S}_{j}^{\perp}$ is the identity matrix in all columns except the $j$ th column, where it has -1 as a diagonal entry and 2 for all off-diagonal entries. The group $\mathcal{A}_{n}^{\perp}$ is a group of integer matrices in all dimensions $n$. It is straightforward to check that $\mathcal{A}_{n}^{\perp} \subset \operatorname{Aut}\left(Q_{D, n}\right)$. It is evident that $\left(\mathbf{S}_{j}^{\perp}\right)^{2}=I$ holds for all $j$. The geometric interpretation of the operation $\mathbf{S}_{j}^{\perp}$ on a Descartes configuration $\mathcal{D}$ is that it encodes inversion with respect to the $i$ th sphere of that configuration.

Theorem 4.2. The dual Apollonian group $\mathcal{A}_{n}^{\perp}$ is a discrete subgroup of $G L(n+2, \mathbb{R})$ for all $n \geq 2$.

Proof. This holds since $\mathcal{A}_{n}^{\perp}$ is a subgroup of the discrete group $G L(n+2, \mathbb{Z})$.

Orbits of this group, acting on Descartes configurations, retain a "packing" property at the level of individual spheres.

Theorem 4.3. For all $n \geq 2$, an orbit of the dual Apollonian group $\mathcal{A}_{n}^{\perp}$ acting on a single Descartes configuration gives a "packing" of spheres in the weak sense that no two spheres in distinct Descartes configurations of the orbit cross each other, i.e. any two such spheres either coincide, or are disjoint or are tangent.

Proof. This can be seen geometrically by constructing the dual packing starting from a single Descartes configuration $\mathcal{D}$. The spheres in the resulting "packing" are all nested 
inside spheres of $\mathcal{D}$, and we call the level of a sphere its depth of nesting inside some sphere of $\mathcal{D}$, the spheres in $\mathcal{D}$ being assigned level 0 . One proceeds in stages, where at stage $k-1$ one has the set of Descartes configurations $\left\{\mathbf{S}_{i_{k-1}}^{\perp} \mathbf{S}_{i_{k-2}}^{\perp} \cdots \mathbf{S}_{i_{1}}^{\perp} \mathbf{W}_{\mathcal{D}}\right\}$, in which each $i_{j} \neq i_{j-1}$. We assert as an induction hypothesis, that each stage $k-1$ Descartes configuration has all but one of its spheres at level $k-1$, with one sphere at level $k-2$. This holds for the base case $k=2$ by inspection. At stage $k$ each multiplication by a generator takes a particular Descartes configuration at stage $k-1$ and maps $(n+1)$ of its spheres inside one level $k-1$ sphere of the configuration. (Here the condition $i_{k} \neq i_{k-1}$ is used.) The new Descartes configuration then consists of $(n+1)$ spheres nested to depth $k$ inside $\mathcal{D}$, contained in one outer sphere nested to depth $k-1$. Furthermore, each level $k$ Descartes configuration is nested inside a unique level $(k-1)$-sphere, of which there are $(n+2) \cdot(n+1)^{k-2}$ choices. It follows that the new level $k$ spheres cannot cross any spheres at any levels up to $k-1$, and they also cannot cross any other level $k$ spheres because they are either inside different level $k-1$ spheres, or if they are in the same level $k-1$ sphere, then they form part of a single Descartes configuration. Thus the noncrossing property holds at depth $k$, and the result follows by induction on $k$.

\section{3. n-Dimensional Super-Apollonian Group}

The $n$-dimensional super-Apollonian group $\mathcal{A}_{n}^{S}$ is the group generated by $\mathcal{A}_{n}$ and $\mathcal{A}_{n}^{\perp}$, so that

$$
\mathcal{A}_{n}^{\perp}=\left\langle\mathbf{S}_{1}, \mathbf{S}_{2}, \ldots, \mathbf{S}_{n+2}, \mathbf{S}_{1}^{\perp}, \mathbf{S}_{2}^{\perp}, \ldots, \mathbf{S}_{n+2}^{\perp}\right\rangle .
$$

This group consists of integer matrices when $n=2$ or 3 , and of rational matrices otherwise. In particular, $\mathcal{A}_{n}^{S}$ is a discrete subgroup of $G L(n+2, \mathbb{R})$ for $n=2$ or $n=3$, and is not a discrete subgroup for $n \geq 4$. The property that some spheres in different Descartes configurations intersect nontangentially when $n \geq 4$ is inherited from the action of the Apollonian group.

We do not know if the super-Apollonian group is a Coxeter group. We do know that its generators satisfy the Coxeter relations given in the following lemma. It seems plausible that for $n \geq 4$ these are a generating set of relations; if so, then $\mathcal{A}_{n}^{S}$ would be a hyperbolic Coxeter group.

Theorem 4.4. The super-Apollonian group $\mathcal{A}_{n}^{S}=\left\langle\mathbf{S}_{1}, \mathbf{S}_{2}, \ldots, \mathbf{S}_{n+2}, \mathbf{S}_{1}^{\perp}, \mathbf{S}_{2}^{\perp}, \ldots, \mathbf{S}_{n+2}^{\perp}\right\rangle$ has generators satisfying the relations.

$$
\mathbf{S}_{j}^{2}=I \quad \text { and } \quad\left(\mathbf{S}_{j}^{\perp}\right)^{2}=I, \quad 1 \leq j \leq n+2,
$$

and

$$
\mathbf{S}_{j} \mathbf{S}_{k}^{\perp}=\mathbf{S}_{k}^{\perp} \mathbf{S}_{j} \quad \text { when } \quad j \neq k .
$$

Proof. This is a direct calculation from the definition. To appreciate (4.4) one can consider more generally $(n+2) \times(n+2)$ matrices $\mathbf{S}_{j}(\lambda)$ whose entries are the identity matrix except in the $j$ th row, where they are -1 on the diagonal and $\lambda$ off the diagonal. 
Then $\mathbf{S}_{j}(\lambda)^{2}=I$ holds for $1 \leq j \leq n$, but the extra relations (4.4) hold only when $\lambda=2 /(n-1)$. (Compare the $(j, k)$ th entry of the products $\mathbf{S}_{j} \mathbf{S}_{k}^{\perp}$ and $\mathbf{S}_{k}^{\perp} S_{j}$.) Note however that $\mathbf{S}_{j}(\lambda) \in \operatorname{Aut}\left(Q_{D, n}\right)$ if and only if $\lambda=2 /(n-1)$.

As mentioned above for the Apollonian group, there is a discrete action of the superApollonian group in certain dimensions, given by its diagonal action of $\mathcal{A}_{n}^{S}$ on the product parameter space $\mathcal{M}_{\mathbb{D}}^{n}(\mathbb{R}) \times \prod_{p \mid n^{*}} \mathcal{M}_{\mathbb{D}}^{n}\left(\mathbb{Q}_{p}\right)$, provided that all the spaces $\mathcal{M}_{\mathbb{D}}^{n}\left(\mathbb{Q}_{p}\right)$ are nonempty. As shown in Section 6.2, this will be the case when $n=2 k^{2}$ or $(2 k+1)^{2}$, for some $k \geq 1$.

\subsection{Apollonian Cluster Ensembles}

In the two-dimensional case Apollonian packings could be described as (a) a collection of circles, or (b) the orbit of four circles under a certain discrete group of Möbius transformations or (c) the Descartes configurations given by an orbit of the Apollonian group. The first two of these notions do not generalize to all dimensions, but version (c) does.

Definition 4.1. An Apollonian cluster ensemble in $n$ dimensions is defined to be the orbit of the Apollonian group $\mathcal{A}_{n}$ of a given ordered, oriented Descartes configuration $\mathcal{D}_{0}$ in the parameter space $\mathcal{M}_{\mathbb{D}}^{n}$.

This object can be viewed as a discrete set in the parameter space $\mathcal{M}_{\mathbb{D}}^{n}$ of all (ordered, oriented) Descartes configurations in dimension $n$. In dimension $n=3$ the spheres in an Apollonian cluster ensemble yield a sphere packing. However, in dimensions $n \geq 4$ the spheres in any such ensemble overlap and no longer correspond to a packing, as shown by Lemma 4.1. Because the Apollonian group consists of rational matrices, we can ask if there are $n$-dimensional Apollonian cluster ensembles with rationality properties, either of their curvatures or of their full augmented curvature-center coordinate matrices $\mathbf{W}_{\mathcal{D}}$.

Similarly one can define a dual Apollonian cluster ensemble to be an orbit of the dual Apollonian group $\mathcal{A}_{n}^{\perp}$ of a single Descartes configuration. It is necessarily a discrete set, because $\mathcal{A}_{n}^{\perp}$ has an integral structure, so the identity matrix is isolated in the group $\mathcal{A}_{n}^{\perp}$. We can ask about rationality or integrality properties of orbits of this group.

A super-Apollonian cluster ensemble is an orbit of the super-Apollonian group $\mathcal{A}_{n}^{S}$. We can ask about rationality properties of orbits of this group, and integrality properties in dimensions $n=2$ and $n=3$. For $n=2$ these were answered in Part II, and for $n=3$ we show in Section 6 that the integral $\mathbf{W}_{\mathcal{D}}$ does not exist. For $n \geq 4$ these orbits are not discrete, but in certain dimensions can have a rational structure inherited from the super-Apollonian group, see Section 6.

\section{Presentation for the $\boldsymbol{n}$-Dimensional Apollonian Group}

In [18] we obtained a presentation for the super-Apollonian group $\mathcal{A}_{2}^{S}$ in dimension 2 , which established that it was a hyperbolic Coxeter group. Theorem 4.4 gave some 
nontrivial relations in the super-Apollonian group $\mathcal{A}_{n}^{S}$, all of Coxeter type. There are additional relations, at least in dimension 3, see Theorem 5.1. It may well be that $\mathcal{A}_{n}^{S}$ is a hyperbolic Coxeter group for all $n \geq 3$. However, it seems a complicated problem to determine a presentation of $\mathcal{A}_{n}^{S}$ in general, and here we establish a more limited result.

We determine a presentation for the Apollonian group $\mathcal{A}_{n}$ in dimensions $n \geq 3$, given in the next two results. In all cases it is a hyperbolic Coxeter group.

Theorem 5.1. For dimension $n=3$ the Apollonian group $\mathcal{A}_{3}=\left\langle\mathbf{S}_{1}, \mathbf{S}_{2}, \mathbf{S}_{3}, \mathbf{S}_{4}, \mathbf{S}_{5}\right\rangle$ has the relations $\mathbf{S}_{j}^{2}=\mathbf{I}$ for $1 \leq j \leq 5$ and the additional relations

$$
\left(\mathbf{S}_{j} \mathbf{S}_{k}\right)^{3}=\mathbf{I}, \quad \text { when } \quad j \neq k .
$$

These are a generating set of relations, and $\mathcal{A}_{3}$ is a hyperbolic Coxeter group.

Proof. Recall that

$$
\mathbf{S}_{1}=\left[\begin{array}{rrrrr}
-1 & 1 & 1 & 1 & 1 \\
0 & 1 & 0 & 0 & 0 \\
0 & 0 & 1 & 0 & 0 \\
0 & 0 & 0 & 1 & 0 \\
0 & 0 & 0 & 0 & 1
\end{array}\right]
$$

and the other $\mathbf{S}_{j}$ are permutations of the $j$ th and first rows and columns of $\mathbf{S}_{1}$. It is easy to check that the generators satisfy all the relations given above; in what follows we denote this set of relations $\mathcal{R}$. We note that relations (5.1) can be written in either of the forms $\left(\mathbf{S}_{j} \mathbf{S}_{k}\right)^{2}=\mathbf{S}_{k} \mathbf{S}_{j}$ or $\mathbf{S}_{j} \mathbf{S}_{k} \mathbf{S}_{j}=\mathbf{S}_{k} \mathbf{S}_{j} \mathbf{S}_{k}$.

We now consider a general word $U=\mathbf{U}_{1} \mathbf{U}_{2} \cdots \mathbf{U}_{n}$ with each $\mathbf{U}_{j}=\mathbf{S}_{i_{j}}$, where inverses of generators are eliminated by relations $\mathbf{S}_{i}^{-1}=\mathbf{S}_{i}$. A subword of $\mathbf{U}$ is any word $\mathbf{U}_{j} \mathbf{U}_{j+1} \cdots \mathbf{U}_{k}$. We call a word reduced if it has the following properties:

(i) 2-reduced. It contains no subword of form $\mathbf{S}_{j} \mathbf{S}_{j}$.

(ii) B-reduced. It contains no subword of form $\mathbf{V}_{1} \mathbf{V}_{2} \cdots \mathbf{V}_{2 m}$ in which $\mathbf{V}_{1}=\mathbf{V}_{3}$, $\mathbf{V}_{2 j}=\mathbf{V}_{2 j+3}$ for $1 \leq j \leq m-2$, and $\mathbf{V}_{2 m-2}=\mathbf{V}_{2 m}$.

Conditions (i) and (ii) together allow the refinement of (ii) to assert $\mathbf{V}_{1}=\mathbf{V}_{3} \neq \mathbf{V}_{2}$, $\mathbf{V}_{2 j}=\mathbf{V}_{2 j+3} \neq \mathbf{V}_{2 j+2}$ and $\mathbf{V}_{2 m-2}=\mathbf{V}_{2 m} \neq \mathbf{V}_{2 m-1}$. Also the case $m=2$ rules out words $\mathbf{S}_{j} \mathbf{S}_{k} \mathbf{S}_{j} \mathbf{S}_{k}$. The definition also implies: every subword of a $B$-reduced word is also $B$-reduced.

We assert that every nonreduced word can be simplified to a reduced word of a shorter length, using the relations $\mathcal{R}$. If (i) is violated, then using the relation $\mathbf{S}_{j}^{2}=I$ we may replace the subword by the empty word, shortening it by two. If (ii) holds then we can replace the subword by $\mathbf{V}_{2} \mathbf{V}_{3} \cdots \mathbf{V}_{2 m-1}$, decreasing its length by two. This reduction is done by a sequence of replacements of the form $\mathbf{S}_{j} \mathbf{S}_{k} \mathbf{S}_{j}$ by $\mathbf{S}_{k} \mathbf{S}_{j} \mathbf{S}_{k}$ applied successively at positions 1, 3, ., 2m-3, which ends with $\mathbf{V}_{2}$ at the beginning and $\mathbf{V}_{2 m-1} \mathbf{V}_{2 m} \mathbf{V}_{2 m}$ at the end. Then the final letters $\mathbf{V}_{2 m} \mathbf{V}_{2 m}$ are deleted by rule (i), achieving a shortening by two. Starting with any word $\mathbf{U}$ and applying this reduction process, we eventually arrive at a reduced word or the empty word. 
The theorem is equivalent to the assertion that each nonempty reduced word is not the identity. For if the relations $\mathcal{R}$ did not generate all relations, there would exist another relation, necessarily forcing some nonempty reduced word to be the identity.

We introduce some (matrix) invariants associated to a word in the generators. Let $\mathbf{e}_{j}$ be the $j$ th unit (column) vector, i.e. the $j$ th column of the $5 \times 5$ identity matrix, and set $\mathbf{1}_{5}=(1,1,1,1,1)^{T}$. We define

$$
\sigma_{j}(\mathbf{U}):=\mathbf{e}_{j}^{T} \mathbf{U} \mathbf{1}_{5},
$$

the sum of entries in row $j$ of $\mathbf{U}$, and

$$
\Sigma(\mathbf{U}):=\mathbf{1}_{5}^{T} \mathbf{U} \mathbf{1}_{5},
$$

the sum of all entries of $\mathbf{U}$, which we call its mass.

We compute the effect on these invariants of multiplying by a generator. The matrix $\mathbf{S}_{j} \mathbf{U}$ has all rows but the $j$ th row the same as $\mathbf{U}$, with its $j$ th row equal to the sum of all rows of $\mathbf{U}$ minus twice its $j$ th row, which yields

$$
\sigma_{j}\left(\mathbf{S}_{j} \mathbf{U}\right)=\Sigma(\mathbf{U})-2 \sigma_{j}(\mathbf{U}),
$$

and $\sigma_{k}\left(\mathbf{S}_{j} \mathbf{U}\right)=\sigma_{k}(\mathbf{U})$ if $k \neq j$. It also gives

$$
\Sigma\left(\mathbf{S}_{j} \mathbf{U}\right)=2 \Sigma(\mathbf{U})-3 \sigma_{j}(\mathbf{U}) .
$$

Now, we define

$$
\delta_{j}(\mathbf{U}):=\Sigma\left(\mathbf{S}_{j} \mathbf{U}\right)-\Sigma(\mathbf{U}) .
$$

This quantity measures the increase in the total mass of a matrix when we multiply by $\mathbf{S}_{j}$. Two key properties of this measure are that if $j \neq k$ then

$$
\delta_{j}\left(\mathbf{S}_{k} \mathbf{U}\right)=\delta_{j}(\mathbf{U})+\delta_{k}(\mathbf{U})
$$

while if $j=k$ then

$$
\delta_{j}\left(\mathbf{S}_{j} \mathbf{U}\right)=-\delta_{j}(\mathbf{U}) .
$$

To verify these, first observe that

$$
\delta_{j}(\mathbf{U})=\left(2 \Sigma(\mathbf{U})-3 \sigma_{j}(\mathbf{U})\right)-\Sigma(\mathbf{U})=\Sigma(\mathbf{U})-3 \sigma_{j}(\mathbf{U}) .
$$

Therefore

$$
\begin{aligned}
\delta_{j}\left(\mathbf{S}_{k} \mathbf{U}\right) & =\Sigma\left(\mathbf{S}_{k} \mathbf{U}\right)-3 \sigma_{j}\left(\mathbf{S}_{k} \mathbf{U}\right) \\
& =2 \Sigma(\mathbf{U})-3 \sigma_{k}(\mathbf{U})-3 \sigma_{j}(\mathbf{U}) \\
& =\Sigma(\mathbf{U})-3 \sigma_{j}(\mathbf{U})+\Sigma(\mathbf{U})-3 \sigma_{k}(\mathbf{U}) \\
& =\delta_{j}(\mathbf{U})+\delta_{k}(\mathbf{U})
\end{aligned}
$$


which gives (5.8). Similarly, using (5.7),

$$
\begin{aligned}
\delta_{j}\left(\mathbf{S}_{j} \mathbf{U}\right) & =\Sigma\left(\mathbf{S}_{j} \mathbf{S}_{j} \mathbf{U}\right)-\Sigma\left(\mathbf{S}_{j} \mathbf{U}\right) \\
& =-\left(\Sigma\left(\mathbf{S}_{j} \mathbf{U}\right)-\Sigma(\mathbf{U})\right) \\
& =-\delta_{j}(\mathbf{U}) .
\end{aligned}
$$

One consequence of these two properties is that

$$
\delta_{j}\left(\mathbf{S}_{k} \mathbf{U}\right)=\delta_{k}\left(\mathbf{S}_{j} \mathbf{U}\right) \quad \text { if } \quad j \neq k .
$$

Another consequence is

$$
\delta_{j}\left(\mathbf{S}_{k} \mathbf{S}_{j} \mathbf{U}\right)=\delta_{k}\left(\mathbf{S}_{j} \mathbf{U}\right)+\delta_{j}\left(\mathbf{S}_{j} \mathbf{U}\right)=\left(\delta_{j}(\mathbf{U})+\delta_{k}(\mathbf{U})\right)-\delta_{j}(\mathbf{U})=\delta_{k}(\mathbf{U}) .
$$

We assert that all nonempty reduced words $\mathbf{U}=\mathbf{S}_{j} \mathbf{U}^{\prime}$ have

$$
\delta_{j}\left(\mathbf{U}^{\prime}\right)>0,
$$

so that $\Sigma(\mathbf{U})>\Sigma\left(\mathbf{U}^{\prime}\right)$. If this is proved, then since $\mathbf{U}^{\prime}$ is also reduced, we obtain by induction on the length of $\mathbf{U}$ that

$$
\Sigma(\mathbf{U}) \geq \Sigma\left(\mathbf{U}_{n}\right)=7>\Sigma(I)=5,
$$

so that $\mathbf{U}$ cannot be the identity matrix, and the theorem follows.

We establish (5.12) by induction on the length $n$ of $\mathbf{U}=\mathbf{U}_{1} \mathbf{U}_{2} \cdots \mathbf{U}_{n}=\mathbf{U}_{1} \mathbf{U}^{\prime}$, where we suppose $\mathbf{U}_{1}=\mathbf{S}_{j}$. In the base case $n=1$, we consider that $\mathbf{U}^{\prime}=I$, and we then have

$$
\Sigma\left(\mathbf{S}_{j}\right)=7 \geq \Sigma(I)=5,
$$

completing the base case. Now suppose $n \geq 2$ and that the induction hypothesis holds up to $n-1$. We write $\mathbf{U}=\mathbf{S}_{j} \mathbf{S}_{k} \mathbf{U}^{\prime \prime}$ with $\mathbf{U}^{\prime \prime}$ of length $n-2$, noting that $j \neq k$. We are to show $\delta_{j}\left(\mathbf{U}^{\prime}\right)>0$. Now (5.8) gives

$$
\delta_{j}\left(\mathbf{U}^{\prime}\right)=\delta_{j}\left(\mathbf{S}_{k} \mathbf{U}^{\prime \prime}\right)=\delta_{j}\left(\mathbf{U}^{\prime \prime}\right)+\delta_{k}\left(\mathbf{U}^{\prime \prime}\right) .
$$

The induction hypothesis gives $\delta_{k}\left(\mathbf{U}^{\prime \prime}\right)>0$. If $\mathbf{S}_{j} \mathbf{U}^{\prime \prime}$ is reduced, then $\delta_{j}\left(\mathbf{U}^{\prime \prime}\right)>0$ by the induction hypothesis and we are done. So suppose $\mathbf{S}_{j} \mathbf{U}^{\prime \prime}$ is not reduced. Since $\mathbf{U}^{\prime \prime}$ is reduced, a nonreduced subword in it must be an initial segment, which either fails to be 2-reduced or $B$-reduced.

Suppose first that $\mathbf{S}_{j} \mathbf{U}^{\prime \prime}$ is not $B$-reduced, having an initial $B$-word $\mathbf{V}_{1} \cdots \mathbf{V}_{2 m}$ which begins $\mathbf{S}_{j} \mathbf{S}_{l} \mathbf{S}_{j}$ so that $\mathbf{S}_{j} \mathbf{U}^{\prime \prime}=\mathbf{S}_{j} \mathbf{S}_{l} \mathbf{S}_{j} \mathbf{V}$, for some $l \neq j$. We also have $l \neq k$ since $\mathbf{U}^{\prime}=\mathbf{S}_{k} \mathbf{U}^{\prime \prime}$ is 2-reduced. Now we have, using (5.10),

$$
\delta_{j}\left(\mathbf{U}^{\prime}\right)=\delta_{j}\left(\mathbf{S}_{k} \mathbf{U}^{\prime \prime}\right)=\delta_{k}\left(\mathbf{S}_{j} \mathbf{U}^{\prime \prime}\right) .
$$

Applying the $B$-reduction procedure to the initial segment $\mathbf{V}_{1} \cdots \mathbf{V}_{2 m}$ simplifies the word $\mathbf{S}_{j} \mathbf{U}^{\prime \prime}$ to a word $\mathbf{S}_{l} \mathbf{S}_{j} \mathbf{V}^{\prime}$ that is shorter by two letters than $\mathbf{S}_{j} \mathbf{U}^{\prime \prime}$, but is equal to it as a matrix. It follows using (5.14) that

$$
\delta_{k}\left(\mathbf{S}_{l} \mathbf{S}_{j} \mathbf{V}^{\prime}\right) \equiv \delta_{k}\left(\mathbf{S}_{j} \mathbf{U}^{\prime \prime}\right)=\delta_{j}\left(\mathbf{U}^{\prime}\right) .
$$


We now assert that $\mathbf{S}_{l} \mathbf{S}_{j} \mathbf{V}^{\prime}$ itself is reduced. To see this, write $\mathbf{S}_{l} \mathbf{S}_{j} \mathbf{V}^{\prime}=\mathbf{V}_{2} \mathbf{V}_{3} \cdots \mathbf{V}_{2 m-1} \mathbf{V}^{\prime \prime}$ with $\mathbf{V}^{\prime \prime}=\mathbf{S}_{r} \mathbf{S}_{t} \mathbf{V}^{\prime \prime \prime}$. Since $\mathbf{U}^{\prime \prime}=\mathbf{S}_{j} \mathbf{V}^{\prime}$ was reduced, any further reduction involves a subword $\mathbf{X}$ that contains $\mathbf{V}_{2 m-2} \mathbf{S}_{r}$. Now $\mathbf{S}_{r} \neq \mathbf{V}_{2 m}$ or else $\mathbf{U}^{\prime \prime}$ includes $\mathbf{V}_{2 m} \mathbf{S}_{r}=\mathbf{V}_{2 m} \mathbf{V}_{2 m}$ contradicting $\mathbf{U}^{\prime \prime}$ being reduced. Next $\mathbf{S}_{r} \neq \mathbf{V}_{2 m-1}$, for otherwise $\mathbf{U}^{\prime \prime}$ would contain the subword $\mathbf{V}_{2 m-2} \mathbf{V}_{2 m-1} \mathbf{V}_{2 m} \mathbf{V}_{2 m-1}$ which is a $B$-word since $\mathbf{V}_{2 m}=\mathbf{V}_{2 m-2}$, contradicting $\mathbf{U}^{\prime \prime}$ being reduced. It follows that $\mathbf{V}_{2 m-2} \mathbf{V}_{m-1} \mathbf{S}_{r}=\mathbf{V}_{2 m} \mathbf{V}_{2 m-1} \mathbf{S}_{r}$ consists of three distinct letters (generators). Thus any nonreduced subword $\mathbf{X}=\mathbf{X}^{\prime} \mathbf{V}_{2 m} \mathbf{V}_{2 m-1} \mathbf{S}_{r} \mathbf{X}^{\prime \prime}$ inside $\mathbf{S}_{l} \mathbf{S}_{j} \mathbf{V}^{\prime}$ must be a $B$-word. However, then $\mathbf{U}^{\prime \prime}$ contains the $B$-word $\mathbf{V}_{2 m} \mathbf{V}_{2 m-1} \mathbf{V}_{2 m} \mathbf{S}_{r} \mathbf{X}^{\prime \prime}$, contradicting $\mathbf{U}^{\prime \prime}$ being reduced. We conclude that $\mathbf{S}_{l} \mathbf{S}_{j} \mathbf{V}^{\prime}$ is reduced. We deduce that $\mathbf{S}_{k} \mathbf{S}_{l} \mathbf{S}_{j} \mathbf{V}^{\prime}$ is reduced, because $\mathbf{S}_{l} \mathbf{S}_{j} \mathbf{V}^{\prime}$ is reduced and $\mathbf{S}_{k}$ differs from both of its first two letters. The induction hypothesis now applies to give $\delta_{k}\left(\mathbf{S}_{l} \mathbf{S}_{j} \mathbf{V}^{\prime}\right)>0$, which with (5.15) gives $\delta_{j}\left(\mathbf{U}^{\prime}\right)>0$.

The remaining case is that when $\mathbf{S}_{j} \mathbf{U}^{\prime \prime}$ is not 2-reduced. Then $\mathbf{U}^{\prime \prime}=\mathbf{S}_{j} \mathbf{V}$ and $\mathbf{U}=$ $\mathbf{S}_{j} \mathbf{S}_{k} \mathbf{S}_{j} \mathbf{V}$. We have then, by (5.11), that

$$
\delta_{j}\left(\mathbf{U}^{\prime}\right)=\delta_{k}(\mathbf{V}) .
$$

If $\mathbf{S}_{k} \mathbf{V}$ is reduced, then the induction hypothesis gives $\delta_{k}(\mathbf{V})>0$, which gives the desired result. If it is not reduced, then it is either not 2-reduced or $B$-reduced. If it is not 2reduced, then we have $\mathbf{V}=\mathbf{S}_{k} \mathbf{V}^{\prime}$, in which case the original word $\mathbf{U}=\mathbf{S}_{j} \mathbf{S}_{k} \mathbf{S}_{j} \mathbf{S}_{k} \mathbf{V}^{\prime}$ is not $B$-reduced, contradicting the hypothesis. If $\mathbf{S}_{k} \mathbf{V}$ is not $B$-reduced, then we have $\mathbf{V}=\mathbf{S}_{l} \mathbf{S}_{k} \mathbf{V}^{\prime}$, with $l \neq k$, and $\mathbf{U}=\mathbf{S}_{j} \mathbf{S}_{k} \mathbf{S}_{j} \mathbf{S}_{l} \mathbf{S}_{k} \mathbf{V}^{\prime}$, which shows that $l \neq j$. Now we have by (5.11) again that

$$
\delta_{k}(\mathbf{V})=\delta_{k}\left(\mathbf{S}_{l} \mathbf{S}_{k} \mathbf{V}^{\prime}\right)=\delta_{l}\left(\mathbf{V}^{\prime}\right)
$$

If $\mathbf{S}_{k} \mathbf{V}^{\prime}$ is reduced then the induction hypothesis gives $\delta_{l}\left(\mathbf{V}^{\prime}\right)>0$ which implies that $\delta_{j}\left(\mathbf{U}^{\prime}\right)>0$ as desired. If it is not 2-reduced then $\mathbf{V}^{\prime}=\mathbf{S}_{k} \mathbf{V}^{\prime \prime}$ and this contradicts $\mathbf{U}$ being reduced. There remains the case where $\mathbf{V}^{\prime}$ is not $B$-reduced. Then we get $\mathbf{V}^{\prime}=\mathbf{S}_{m} \mathbf{S}_{l} \mathbf{V}^{\prime \prime}$, with $\delta_{k}\left(\mathbf{V}^{\prime}\right)=\delta_{m}\left(\mathbf{V}^{\prime \prime}\right)$. We can continue recursively in this way until the entire word $\mathbf{U}$ is used up and there are not enough letters to have any $B$-reduced word. We then obtain

$$
\delta_{j}\left(\mathbf{U}^{\prime}\right)=\delta_{k}(\mathbf{V})=\delta_{l}(\mathbf{V})=\cdots=\delta_{n}\left(\mathbf{V}^{(r)}\right)>0 .
$$

This completes the induction step, proving (5.12), and the theorem follows.

Theorem 5.2. For dimension $n \geq 4$ the Apollonian group $\mathcal{A}_{n}=\left\langle\mathbf{S}_{1}, \mathbf{S}_{2}, \ldots, \mathbf{S}_{n+2}\right\rangle$ is a hyperbolic Coxeter group whose only relations are

$$
\mathbf{S}_{j}^{2}=\mathbf{I}_{n+2} \quad \text { for } \quad 1 \leq j \leq n+2 .
$$

Proof. Suppose $n \geq 4$. We must show that no nonempty product of the $\mathbf{S}_{j}$ 's, with distinct adjacent terms, is equal to the identity matrix $I_{n+2}$.

Let $g_{n}=1 /(n-1)$ and let $\mathbf{A}_{j}=\frac{1}{2}\left(\mathbf{S}_{j}-\mathbf{I}_{n+2}\right)$ so that $\mathbf{S}_{j}=\mathbf{I}_{n+2}+2 \mathbf{A}_{j}$. Then $\mathbf{A}_{j}$ has all entries zero except for the $j$ th row, in which all entries are $g_{n}$ except for the $j$ th element, which is -1 . Let

$$
\mathbf{A}=g_{n} \mathbf{1}_{n+2} \mathbf{1}_{n+2}^{T}-n \mathbf{I}_{n+2}
$$


be the matrix with -1 on the main diagonal and $g_{n}$ elsewhere, so that its $(i, j)$ element is

$$
a(i, j)= \begin{cases}-1, & \text { if } \quad i=j \\ g_{n}, & \text { if } \quad i \neq j .\end{cases}
$$

Then $\mathbf{A}_{j}$ and $\mathbf{A}$ both have $j$ th row $(a(j, 1), \ldots, a(j, n+2))$. We note that $\mathbf{A}$ is nonsingular.

Suppose that $k \geq 2$, that $1 \leq j_{1}, \ldots, j_{k} \leq n+2$, that no two consecutive $j_{r}$ 's are equal, and that $S$ is the product $S=\mathbf{S}_{j_{1}} \mathbf{S}_{j_{2}} \cdots \mathbf{S}_{j_{k}}$. We assume that $\mathbf{S}=\mathbf{I}_{n+2}$ and derive a contradiction. We have

$$
\begin{aligned}
\mathbf{S}-\mathbf{I}_{n+2}= & \mathbf{S}_{j_{1}} \mathbf{S}_{j_{2}} \cdots \mathbf{S}_{j_{k}}-\mathbf{I}_{n+2} \\
= & \left(\mathbf{I}_{n+2}+2 \mathbf{A}_{j_{1}}\right) \cdots\left(\mathbf{I}_{n+2}+2 \mathbf{A}_{j_{k}}\right)-\mathbf{I}_{n+2} \\
= & 2 \sum_{1 \leq r \leq k} \mathbf{A}_{j_{r}}+4 \sum_{1 \leq r<s \leq k} \mathbf{A}_{j_{r}} \mathbf{A}_{j_{s}} \\
& +8 \sum_{1 \leq r<s<t \leq k} \mathbf{A}_{j_{r}} \mathbf{A}_{j_{s}} \mathbf{A}_{j_{t}}+\cdots+2^{k} \mathbf{A}_{j_{1}} \cdots \mathbf{A}_{j_{k}} .
\end{aligned}
$$

The $\mathbf{A}_{j}$ 's multiply more simply than the $\mathbf{S}_{j}$ 's, compensating for the more complicated expression involving them. A product like $8 \mathbf{A}_{j_{r}} \mathbf{A}_{j_{s}} \mathbf{A}_{j_{t}}$, which we call an Aproduct, has one nonzero row, row $j_{r}$, which is equal to row $j_{t}$ of $\mathbf{A}$ multiplied by $8 a\left(j_{r}, j_{s}\right) a\left(j_{s}, j_{t}\right)$. The terms of the latter product are -1 or $g-n$, depending on whether or not $j_{r}=j_{s}$ and $j_{s}=j_{t}$. We call the scalar $8 a\left(j_{r}, j_{s}\right) a\left(j_{s}, j_{t}\right)$ the $a$ product corresponding to the $A$-product $8 \mathbf{A}_{j_{r}} \mathbf{A}_{j_{s}} \mathbf{A}_{j_{t}}$. In general, an $a$-product has the form $\pm 2^{\alpha} g_{n}^{\beta}, \alpha>\beta$.

The $j$ th row of $\mathbf{S}-\mathbf{I}_{n+2}$ gets a contribution from each $A$-product that starts with $\mathbf{A}_{j}$. It is a linear combination of rows of $\mathbf{A}$ determined by the last subscript in each contributing $A$-product. Because this linear combination is zero, each of its coefficients must be zero, since $\mathbf{A}$ is full rank. Therefore, for each $t$ and $u$ in $\left\{j_{1}, \ldots, j_{k}\right\}$, the sum of all the $A$-products corresponding to $A$-products that start with $\mathbf{A}_{t}$ and end with $\mathbf{A}_{u}$ must be zero. This sum is a polynomial in the variable $g=g_{n}$, call it $P_{t, u}(g)$. Because $A$-products have the form $\pm 2^{\alpha} g^{\beta}, \alpha>\beta$, the coefficient of $g_{n}^{i}$ in this polynomial is divisible by $2^{i+1}$, so we may write

$$
P_{t, u}(g)=\sum_{i=0}^{k-1} c_{t, u, i} 2^{i+1} g^{i}
$$

for integers $c_{t, u, i}$.

Now consider $P_{j_{1}, j_{k}}(g)$. This has degree $k-1$. In fact, the only $A$-product that can contribute an $a$-product of degree $k-1$ is $2^{k} \mathbf{A}_{j_{1}} \cdots \mathbf{A}_{j_{k}}$, and since successive $\mathbf{A}_{j_{r}}$ 's are distinct, the corresponding $a$-product is $2^{k} g_{n}^{k-1}$, i.e. $c_{j_{1}, j_{k}, k-1}=1$. Therefore, by the Rational Root Theorem, since $g_{n}=1 /(n-1)$ is a root of $P_{j_{1}, j_{k}}(g)$, we must either have $n-1$ dividing $2^{k}$, or $n=2^{m}+1$ for some $m>1$, in which case $g_{n}=1 / 2^{m}$. Writing $c_{i}=c_{j_{1}, j_{k}, i}$ we then have

$$
P_{j_{1}, j_{k}}\left(g_{n}\right)=\sum_{i=0}^{k-1} c_{i} 2^{i+1} g_{n}^{i}=0
$$


On multiplying by $2^{m(k-1)-k}$ this yields the equation

$$
\sum_{i=0}^{k-1} c_{i} 2^{(m-1)(k-1-i)}=0
$$

with integer entries $c_{i}$. We now check that this equation is impossible (mod 2). All terms except the last are even since $(m-1)(k-1-i)>0$ when $0 \leq i \leq k-2$. The $(k-1)$ st term in the last sum is $c_{k-1} 2^{0}=1$, so we get $0 \equiv 1(\bmod 2)$, the desired contradiction. We conclude that $\mathbf{S} \neq \mathbf{I}_{n+2}$, and the theorem follows.

\section{6. $\quad S$-Integral Apollonian Cluster Ensembles (Dimension $n$ )}

We study integrality and rationality properties for Apollonian cluster ensembles. Given a finite set of primes $S$, we say that a rational number is $S$-integral if its denominator is divisible only by powers of primes in $S$. By convention we let $S=1$ denote the case when there are no primes in $S$.

The Apollonian group consists of integer matrices in dimensions 2 and 3 , and retains an $S$-integral structure in all dimensions, for suitable $S$. In each dimension $n$ we consider the questions:

(1) Does there exist some $S$ and an Apollonian cluster ensemble all of whose Descartes configurations consist of spheres having $S$-integral curvatures (" $S$-integral ensemble")?

(2) Does there exist some $S$ and an Apollonian cluster ensemble all of whose Descartes configurations have augmented curvature-center coordinate matrices $\mathbf{W}_{\mathcal{D}} S$-integral ("super $S$-integral ensemble")?

We show that $S$-integral ensembles exist in all dimensions, if $S$ is chosen properly. However, we show that super $S$-integral ensembles can exist only in dimensions $n=2 m^{2}$ or $n=(2 m+1)^{2}$, for integer $m$, again with $S$ chosen appropriately.

\subsection{S-Integral Apollonian Cluster Ensembles}

We say that an Apollonian cluster ensemble is $S$-integral if the curvature of every sphere in the ensemble is $S$-integral.

Theorem 6.1. In each dimension $n \geq 2$ there exists an S-integral Apollonian cluster ensemble in which $S$ is specified as:

(1) $S$ is the set of primes dividing $n-1$ if $n$ is even.

(2) $S$ is the set of primes dividing $(n-1) / 2$ if $n$ is odd.

Proof. It suffices to show that the Descartes equation

$$
\mathbf{b}^{T} \mathbf{Q}_{D, n} \mathbf{b}=0
$$

has a nonzero $S$-integral solution $\mathbf{b}$ for each $n \geq 2$. There is such a configuration $\mathcal{D}$ which is not only $S$-integral, but integral, with curvatures $(0,0,1,1, \ldots, 1)$. It consists of two 
parallel hyperplanes separated by distance 2 together with $n$ unit spheres whose centers comprise the vertices and an $(n-1)$-dimensional simplex in a hyperplane parallel to the two hyperplanes in the configuration, and lying midway between them.

The other Descartes configurations in the Apollonian cluster ensemble and superApollonian cluster ensemble generated by this configuration are $S$-integral, where $S$ is the set of primes dividing the denominator of $2 /(n-1)$, since they have associated matrices $\mathbf{U} \mathbf{W}_{\mathcal{D}}$ for some $\mathbf{U}$ in the Apollonian group.

In dimension 2 we can take $S=1$, as we saw in [19]. In that case an Apollonian cluster ensemble consists of the Descartes configurations in an Apollonian circle packing. In [20] we studied, in various number-theoretic questions related to the integer, curvatures appearing in integer Apollonian circle packings.

\subsection{Super-S-Integral Apollonian Cluster Ensembles}

We say that a Descartes configuration $\mathcal{D}$ is super-S-integral if its augmented curvaturecenter coordinate matrix $\mathbf{W}_{\mathcal{D}}$ is $S$-integral. Similarly, an Apollonian cluster ensemble is super-S-integral if every Descartes configuration $\mathcal{D}$ in the packing has an $S$-integral augmented curvature-center matrix. The next lemma reduces the question of super- $S$ integrality of an Apollonian cluster ensemble to that of a single Descartes configuration.

Theorem 6.2. If a single Descartes configuration is super-S-integral, then the Apollonian cluster ensemble it generates is super- $S^{\prime}$-integral, where $S^{\prime}$ consists of $S$ together with all primes dividing the denominator of $2 /(n-1)$.

Proof. This follows from the fact that the Apollonian group consists of rational matrices whose entries have denominators that are divisible only by primes dividing the denominator of $2 /(n-1)$, when put in lowest terms.

It seems to be a difficult problem to determine, for specific $S$, for which dimensions there might exist a super- $S$-integral Descartes configuration. We consider the weaker question of whether in a given dimension there exists a super- $S$-integral Descartes configurations for some $S$. This is the same as the existence of Descartes configurations $\mathcal{D}$ having a rational augmented curvature-center matrix $\mathbf{W}_{\mathcal{D}}$, and we call such Descartes configurations super-rational.

According to the Augmented Euclidean Descartes Theorem (Theorem 3.1), superrational Descartes configurations occur exactly in those dimensions $n$ in which there exists an invertible rational matrix $\mathbf{W}$ such that

$$
\mathbf{W}^{T} \mathbf{Q}_{D, n} \mathbf{W}=\mathbf{Q}_{W, n}:=\left[\begin{array}{rrc}
0 & -4 & 0 \\
-4 & 0 & 0 \\
0 & 0 & 2 I_{n}
\end{array}\right],
$$

that is, the quadratic form $Q_{D, n}$ is rationally equivalent to the form $Q_{W, n}$. We use this fact to determine in which dimensions such configurations exist. 
Theorem 6.3. A necessary and sufficient condition on the dimension $n$ for a super-Sintegral Descartes configuration to exist for some $S$ is that $n=2 k^{2}$ or $(2 k-1)^{2}$ for some positive integer $k$.

To establish this result, we proceed in a series of lemmas.

Lemma 6.4. Given a Descartes configuration $\mathcal{D}$ in $\mathbb{R}^{n}$ its associated augmented matrix $\mathbf{W}_{\mathcal{D}}$ has

$$
\operatorname{det}\left(\mathbf{W}_{\mathcal{D}}\right)^{2}=n 2^{n+3}
$$

Proof. This follows from taking determinants in (3.12), since the right side has determinant $-2^{n+4}$ while the left side has determinant $\operatorname{det}\left(\mathbf{W}_{\mathcal{D}}\right)^{2} \operatorname{det}\left(\mathbf{Q}_{\mathcal{D}, n}\right)$ and

$$
\operatorname{det}\left(\mathbf{Q}_{\mathcal{D}, n}\right)=-\frac{2}{n}
$$

To verify this last statement, we apply the following row operations to the matrix $\mathbf{Q}_{n}$. Add rows 2 through $n+2$ to the first row, to get a new first row that has all entries $-2 / n$. Then add this row multiplied by $-\frac{1}{2}$ to each of the other rows. Aside from the first row, the first column is zero, and the lower right $(n+1) \times(n+1)$ matrix is the identity. However, this matrix obviously has determinant $-2 / n$.

Lemma 6.5. If a super-rational Descartes configuration exists in dimension $n$, then necessarily $n=2 k^{2}$ or $(2 k-1)^{2}$ for some positive integer $k$.

Proof. A necessary condition for the existence of a Descartes configuration $\mathcal{D}$ whose augmented matrix $\mathbf{W}_{\mathcal{D}}$ has rational entries is that $\operatorname{det}\left(\mathbf{W}_{\mathcal{D}}\right)$ be rational. This requires that $n 2^{n+3}$ be the square of a rational number. By Lemma 6.4, this holds for even $n$ if and only if $n$ is twice a square, and for odd $n$ if and only if $n$ is an (odd) square.

To prove the sufficiency of this condition, we use the theory of equivalence of rational quadratic forms, see [7] or [9]. We write $\mathbf{Q} \simeq_{\mathbb{Q}} \mathbf{Q}^{\prime}$ to mean that the (rational) quadratic form $\mathbf{Q}$ is rationally equivalent to $\mathbf{Q}^{\prime}$. To apply the decision procedure, we first diagonalize $\mathbf{Q}_{n}$ over the rationals, which we do for all $n \geq 2$.

Lemma 6.6. For each $n \geq 2$, the Descartes quadratic form $\mathbf{Q}_{D, n}=\mathbf{I}_{n+2}-$ $(1 / n) \mathbf{1}_{n+2} \mathbf{1}_{n+2}^{T}$ has

$$
\mathbf{Q}_{D, n} \simeq_{\mathbb{Q}} \operatorname{diag}\left(\frac{n-1}{n}, \frac{n-2}{n-1}, \ldots, \frac{2}{3}, 2,2,2,-2\right) .
$$

Proof. We diagonalize the quadratic form as on pp. 92-94 of [9]. Set

$$
M^{(n+2)}:=\mathbf{Q}_{D, n}=\left(x_{0}+y_{0}\right) \mathbf{I}_{n+2}-y_{0} \mathbf{1}_{n+2} \mathbf{1}_{n+2}^{T},
$$


where $x_{0}=(n-1) / n, y_{0}=1 / n$. At the $j$ th stage of reduction we will have

$$
\mathbf{Q}_{D, n} \simeq_{\mathbb{Q}} \operatorname{diag}\left(d_{1}, d_{2}, \ldots, d_{j}, M^{(n+2-j)}\right),
$$

where

$$
\mathbf{M}^{(n+2-j)}=\left(x_{j}+y_{j}\right) \mathbf{I}_{n+2-j}-y_{j} \mathbf{1}_{n+2-j} \mathbf{1}_{n+2-j}^{T}
$$

for certain $x_{j}, y_{j}$. The reduction step is

$$
\left(\mathbf{W}^{(j)}\right)^{T} \mathbf{M}^{(n+2-j)} \mathbf{W}^{(j)}=\operatorname{diag}\left(d_{j+1}, \mathbf{M}^{(n+1-j)}\right) .
$$

To specify $\mathbf{W}^{(j)}$ we first let $\mathbf{W}_{m}(\alpha)$ be the $m \times m$ real matrix

$$
\mathbf{W}_{m}(\alpha)=\left[\begin{array}{cc}
1 & \alpha \cdots \alpha \\
\mathbf{0} & \mathbf{I}_{m-1}
\end{array}\right],
$$

and we set

$$
\mathbf{W}^{(j)}:=\mathbf{W}_{m+2-j}\left(\frac{y_{j}}{x_{j}}\right) .
$$

Substituting this in (6.7), its left side yields a matrix with the form of the right side with

$$
d_{j+1}=x_{j},
$$

and with $x_{j+1}, y_{j+1}$ given by the recursion

$$
\begin{array}{r}
y_{j+1}=y_{j}+\frac{y_{j}^{2}}{x_{j}}, \\
x_{j+1}+y_{j+1}=x_{j}-\frac{y_{j}^{2}}{x_{j}} .
\end{array}
$$

Solving this recursion, by induction on $j$, we obtain

$$
\begin{aligned}
x_{j} & =\frac{n-j-1}{n-j}, & & 0 \leq j \leq n-2, \\
y_{j} & =\frac{1}{n-j}, & & 0 \leq j \leq n-2 .
\end{aligned}
$$

This yields the diagonal elements

$$
d_{j}=\frac{n-j-1}{n-j}, \quad 1 \leq j \leq n-3,
$$

with

$$
\mathbf{Q}_{D, n} \simeq_{\mathbb{Q}} \operatorname{diag}\left(\frac{n-1}{n}, \ldots, \frac{2}{3}, d_{2}, \mathbf{M}^{(4)}\right)
$$


We find $d_{2}=x_{3}=\frac{2}{3}$ and

$$
\mathbf{M}^{(4)}=\left(x_{n-2}+y_{n-2}\right) \mathbf{I}_{4}-y_{n-2} \mathbf{1}_{4} \mathbf{1}_{4}^{T}=\frac{1}{2}\left[\begin{array}{rrrr}
1 & -1 & -1 & -1 \\
-1 & 1 & -1 & -1 \\
-1 & -1 & 1 & -1 \\
-1 & -1 & -1 & 1
\end{array}\right]=\mathbf{Q}_{D, 2} .
$$

For the final step in the reduction we use

$$
N^{T}\left(\mathbf{Q}_{D, 2}\right) N=\operatorname{diag}(2,2,2,-2),
$$

with

$$
N=\left[\begin{array}{rrrr}
1 & -1 & -1 & 1 \\
-1 & 1 & -1 & 1 \\
-1 & -1 & 1 & 1 \\
1 & 1 & 1 & 1
\end{array}\right]
$$

This completes the reduction.

Proof of Theorem 6.3. The necessity of $n=2 k^{2}$ or $(2 k-1)^{2}$ was proved in Lemma 6.5. The sufficiency is equivalent to proving that if $n=2 k^{2}$ and $n=(2 k-1)^{2}$ then

$$
\mathbf{Q}_{D, n} \simeq_{\mathbb{Q}} \mathbf{Q}_{W, n}:=\left[\begin{array}{rrr}
0 & -4 & 0 \\
-4 & 0 & 0 \\
0 & 0 & 2 \mathbf{I}_{n}
\end{array}\right] .
$$

We begin by noting the rational equivalence

$$
\mathbf{Q}_{W, n} \simeq_{\mathbb{Q}} \operatorname{diag}(-2,2, \ldots, 2,2)=\operatorname{diag}\left(-2,2 I_{n+1}\right)
$$

via the matrix

$$
\mathbf{W}_{0}=\frac{1}{2}\left[\begin{array}{rrc}
1 & 1 & 0 \\
1 & -1 & 0 \\
0 & 0 & 2 \mathbf{I}_{n}
\end{array}\right]
$$

By permuting variables we have $\mathbf{Q}_{W, n} \simeq_{\mathbb{Q}} \operatorname{diag}(2,2, \ldots, 2,-2)$. Thus the theorem is equivalent to showing that $\mathbf{Q}_{D, n}$ is rationally equivalent to $\operatorname{diag}(2,2,2, \ldots,-2)$. Lemma 6.6 gives

$$
\begin{aligned}
\mathbf{Q}_{D, n} & \simeq_{\mathbb{Q}}\left(\frac{n-1}{n}, \frac{n-2}{n-1}, \ldots, \frac{3}{2}, 2,2,2,-2\right), \\
& \simeq_{\mathbb{Q}}(n(n-1),(n-1)(n-2), \ldots, 3 \cdot 2,2,2,2,-2),
\end{aligned}
$$

using at the last step a conjugacy by $\mathbf{W}=\operatorname{diag}(n, n-1, \ldots, 2,1,1,1,1)$.

The Hasse-Minkowski theorem says that two rational quadratic forms of the same dimension are equivalent if and only they have the same signature, the ratio of their determinants is a nonzero square and they are $p$-adically equivalent for all primes $p$, see pp. 96ff of [9]. Lemma 6.6 shows that the signatures of $\mathbf{Q}_{D, n}$ and $\operatorname{diag}(2,2,2, \ldots, 2,-2)$ 
agree, and the hypothesis $n=2 k^{2}$ or $n=(2 k-1)^{2}$ is exactly the condition that the ratio of their determinants is a square of a rational number, and it remains to check the $p$-adic invariants.

The $p$-adic invariants $\sigma_{p}(\mathbf{Q})$ are defined $(\bmod 8)$, and for a diagonal form $\mathbf{Q}=$ $\operatorname{diag}\left(d_{1}, d_{2}, \ldots, d_{n}\right)$, one has

$$
\sigma_{p}(\mathbf{Q}) \equiv \sum_{j=1}^{n} \sigma_{p}\left(d_{j}\right) \quad(\bmod 8) .
$$

We recall formulas for $\sigma_{p}(d)$ when $d \in \mathbb{Z}$, see pp. 94-96 of [9]. Write $d=b p^{l}$ with $(b, p)=1$. For $p \geq 3$, and an even power $l=2 j$,

$$
\sigma_{p}(d) \equiv p^{2 j} \equiv 1 \quad(\bmod 8),
$$

while for an odd power $l=2 j+1$,

$$
\sigma_{p}(d) \equiv \begin{cases}p \quad(\bmod 8) & \text { if } \quad(b / p)=1 \\ p+4 \quad(\bmod 8) & \text { if } \quad(b / p)=-1 .\end{cases}
$$

If $p=2$ then for an even power $l=2 j$,

$$
\sigma_{2}(d) \equiv b \quad(\bmod 8)
$$

while for an odd power $l=2 j+1$,

$$
\sigma_{2}(d) \equiv \begin{cases}b & \text { if } \quad b \equiv \pm 1 \quad(\bmod 8) \\ b+4 & \text { if } \quad b \equiv \pm 3 \quad(\bmod 8)\end{cases}
$$

Now (6.14) gives

$$
\sigma_{p}\left(\mathbf{Q}_{D, n}\right) \equiv \sum_{j=0}^{n-3} \sigma_{p}((n-j)(n-j-1))+3 \sigma_{p}(2)+\sigma_{p}(-2) \quad(\bmod 8),
$$

while (6.13) gives

$$
\sigma_{p}\left(\mathbf{Q}_{W, n}\right) \equiv \sum_{j=0}^{n-3} \sigma_{p}(2)+3 \sigma_{p}(2)+\sigma_{p}(-2)(\bmod 8) .
$$

To show equality of these, it suffices to show that for all $p$,

$$
\sum_{j=0}^{n-3} \sigma_{p}(2) \equiv \sum_{j=0}^{n-3} \sigma_{p}((n-j)(n-j-1)) \quad(\bmod 8)
$$

holds whenever $n=2 k^{2}$ or $n=(2 k-1)^{2}$.

Consider first the case that $p \geq 3$ is odd. Then each $\sigma_{p}(2)=1$, so

$$
\sum_{j=0}^{n-3} \sigma_{p}(2) \equiv n-2 \quad(\bmod 8) \text {. }
$$


Now if $p$ does not divide $(n-j)(n-j-1)$ then $\sigma_{p}((n-j)(n-j-1))=1$. The terms divisible by $p$ occur in blocks of two consecutive terms, and we claim that if $p$ divides $j$ then

$$
\sigma_{p}((j+1) j)+\sigma_{p}(j(j-1)) \equiv 2 \quad(\bmod 8) .
$$

Suppose $j=b p^{l}$, where $(b, p)=1$ and $l \geq 1$. If $l$ is even, both terms on the left side of $(6.22)$ are $1(\bmod 8)$ by $(6.16)$, while if $l$ is odd, then if $p \equiv 1(\bmod 4)$, then $(-1 / p)=1$, so the two terms both have values $p$ (resp. $p+4$ ) according as $(b / p)=1$ (resp. -1 ), and their sum is $2 p \equiv 2(\bmod 8)$. If $p \equiv 3(\bmod 4)$, then $(-1 / p)=-1$, so exactly one of $( \pm b / p)$ takes the value -1 , and the two terms add up to $2 p+4 \equiv 2(\bmod 8)$. Thus (6.22) follows. Thus adding up the right side of (6.20) and grouping terms divisible by $p$ in consecutive pairs gives

$$
\sum_{j=0}^{n-3} \sigma_{p}((n-j)(n-j-1)) \equiv \sum_{j=0}^{n-3} 1 \equiv n-2 \quad(\bmod 8) .
$$

There remains an exceptional case where $p \mid n$, in which case $n(n-1)$ is divisible by $p$ and is an unpaired term. Since $n=2 k^{2}$ or $(2 k-1)^{2}$, thus $p^{l} \mid n$ with $l$ even, hence $\sigma_{p}(n(n-1))=1$ in this case, and (6.23) holds. This establishes (6.20) for $p \geq 3$.

Now consider the case $p=2$. Certainly $\sigma_{2}(2)=1$ so (6.21) holds. We claim that

$$
\sigma_{2}((2 j+1) 2 j)+\sigma_{2}(2 j(2 j-1)) \equiv 0 \quad(\bmod 8) .
$$

Write $2 j=2^{l} b$ with $b$ odd, and by checking all possible cases using (6.18) and (6.19), one verifies (6.24). Suppose $n=2 k^{2}$. Then in the right side of (6.20) all terms pair except the first and last, and (6.24) yields

$$
\begin{aligned}
\sum_{j=0}^{n-3} \sigma_{2}((n-j)(n-j-1)) & \equiv \sigma_{2}(n(n-1))+\sigma_{2}(3 \cdot 2) \\
& = \begin{cases}-1+-1 & \text { if } k \equiv 0(\bmod 2) \\
1+-1 & \text { if } k \equiv 1(\bmod 2)\end{cases} \\
& =n-2 \quad(\bmod 8),
\end{aligned}
$$

so $(6.20)$ holds. If $n=(2 k-1)^{2} \equiv 1(\bmod 8)$ then all term pair except the last term, and (6.24) yields

$$
\sum_{j=0}^{n-3} \sigma_{2}((n-j)(n-j-1))=\sigma_{2}(3 \cdot 2) \equiv-1 \quad(\bmod 8),
$$

so (6.20) holds in this case.

Theorem 6.3 establishes the existence of super-rational Descartes configurations in the given dimensions, but does not give a bound for the denominators of the rationals appearing in these configurations. It could be that in certain dimensions $n=2 k^{2}$ and $(2 k+1)^{2}$ there exist strongly integral Descartes configurations, i.e. ones with $S=1$. However, even if such configurations exist for some $n>2$, then the Apollonian cluster ensemble containing them would not inherit the super-integrality property, but only super- $S^{\prime}$-integrality as in Theorem 6.2. We leave this as an open problem. 


\section{7. n-Dimensional Duality Operator}

In the two-dimensional case we showed that there exists a duality operator $\mathbf{D} \in \operatorname{Aut}\left(Q_{D}\right)$ which took each Descartes configuration $\mathcal{D}$ to a new Descartes configuration $\mathcal{D}^{\prime}$ that consists of four circles orthogonal to the original circles, each passing through three of the intersection points of the circles of $\mathcal{D}$. We showed that $\mathbf{D}$ was contained in the normalizer of the super-Apollonian group in two dimensions.

The geometric duality operation based on orthogonal spheres generalizes to higher dimensions as follows. Given $n+1$ mutually tangent spheres in $n$ dimensions, there is a unique sphere through their points of tangency, and this sphere is orthogonal to each of the given $n+1$ spheres, as given in the following (known) result.

Theorem 7.1. Given $n+1$ mutually tangent $(n-1)$-spheres $\left\{C_{i}: 1 \leq j \leq n+1\right\}$ in $\mathbb{R}^{n}$ having disjoint interiors, there exists a unique $(n-1)$-sphere $C^{\perp}$ passing through the $n(n+1) / 2$ tangency points of these spheres. At each such tangency point the normal to the sphere $C^{\perp}$ is perpendicular to the normals of the two spheres $C_{i}$ and $C_{j}$ tangent there.

Proof. The assumption of disjoint interiors (we allow interior to be defined as "exterior" for one sphere) is equivalent to all $n(n+1) / 2$ tangency points of the spheres being distinct. For dimension $n=2$ there is a unique circle through any three distinct points. However, for $n \geq 3$ the conditions are over-determined, since $n+1$ distinct points already determine a unique $(n-1)$-sphere, and the main issue is existence.

Both assertions of the theorem are invariant under Möbius transformations (which preserve angles), and there exists a Möbius transformation taking a set of $n+1$ mutually tangent $(n-1)$-spheres in $\mathbb{R}^{n}$ having disjoint interiors to any other such set, see Theorem 3 of [35]. Thus it suffices to prove the result for a single such configuration, and we consider the configuration of $n+1$ mutually touching spheres of equal radii whose centers are at the vertices of a regular $n$-simplex, and tangency points of the spheres are the midpoints of its edges. The first assertion of the theorem holds in this case because there is an $(n-1)$-sphere whose center is at the center of gravity of this simplex, which passes through the midpoints of every edge of the simplex. Indeed, the isometries preserving an $n$-simplex fix the center of gravity and act transitively on the edges. Note that for $n=2$ the simplex is an equilateral triangle and $C^{\perp}$ is the inscribed circle; however, for $n \geq 3$ the sphere $C^{\perp}$ is neither inscribed nor circumscribed about this simplex.

For the second assertion of the theorem, in this configuration the sphere $C^{\perp}$ has each edge of the $n$-simplex lying in a tangent plane to the sphere; so the normal to $C^{\perp}$ at the midpoint of an edge is perpendicular to that edge. Two spheres $C_{i}$ and $C_{j}$ intersect at the midpoint of an edge, and the normal to their tangent planes points along this edge; thus this normal is perpendicular to the normal to $C^{\perp}$ there.

The second assertion in Theorem 7.1 explains why the sphere $C^{\perp}$ is termed "orthogonal." Thus, given a Descartes configuration of $n+2$ spheres $C_{i}$, we get a system of $n+2$ "orthogonal" spheres

$$
\mathcal{D}^{\perp}:=\left\{C_{1}^{\perp}, \ldots, C_{n+2}^{\perp}\right\}
$$


where $C_{i}^{\perp}$ is associated to the $n+1$ spheres obtained by deleting $C_{i}$. When $n=2$ the new spheres are mutually tangent and give a new Descartes configuration; this gives the "duality" operation D. For $n \geq 3$, however, the spheres are not mutually tangent. In fact, for all $n$ their curvatures satisfy a relation similar in form to the original (twodimensional) Descartes relation, namely,

$$
\sum_{i=1}^{n+2} \frac{1}{r_{i}^{2}}=\frac{1}{2}\left(\sum_{i=1}^{n+2} \frac{1}{r_{i}}\right)^{2},
$$

and not the Soddy-Gossett relation

$$
\sum_{i=1}^{n+2} \frac{1}{r_{i}^{2}}=\frac{1}{n}\left(\sum_{i=1}^{n+2} \frac{1}{r_{i}}\right)^{2},
$$

satisfied by Descartes configurations in $n$-dimensions, see Theorem 1.2 of [23]. (We omit a proof of (7.1).) In particular, for $n \geq 3$ given a Descartes configuration $\mathcal{D}$, the set $\mathcal{D}^{\perp}:=\left\{C_{1}^{\perp}, \ldots, C_{n+2}^{\perp}\right\}$ of orthogonal spheres is not a Descartes configuration.

The question arises, are these $n+2$ "orthogonal" spheres in any special relation to one another? We answer this in terms of an inversive invariant of two arbitrary (not necessarily tangent) oriented spheres.

\section{Definition 7.1.}

(i) The separation between two oriented spheres $C_{1}$ and $C_{2}$ with finite radii $r_{1}$ and $r_{2}$, and with centers distance $d$ apart, is

$$
\Delta\left(C_{1}, C_{2}\right):=\frac{d^{2}-r_{1}^{2}-r_{2}^{2}}{2 r_{1} r_{2}},
$$

provided both spheres are inwardly oriented or outwardly oriented, and is otherwise the negative of the right side of this formula.

(ii) The separation of an oriented sphere $C_{1}$ of finite radius $r_{1}$ and an oriented hyperplane $C_{2}$ is

$$
\Delta\left(C_{1}, C_{2}\right):=\frac{d}{r_{1}},
$$

where $d$ is the (signed) distance from the center $\mathbf{x}_{1}$ of $C_{1}$ to $C_{2}$, measured so that $d \geq 0$ if $\mathbf{x}_{1}$ is not in the interior of $C_{2}$ and $C_{1}$ is inwardly oriented, or if $\mathbf{x}_{1}$ is in the interior of $C_{2}$ and $C_{1}$ is outwardly oriented, and $d<0$ otherwise.

(iii) The separation between two oriented hyperplanes $C_{1}$ and $C_{2}$ is

$$
\Delta\left(C_{1}, C_{2}\right):=-\cos \theta,
$$

where $\theta$ is the dihedral angle between the designated normals at a point of intersection. 
The separation of two spheres is an inversive invariant, hence a Möbius invariant; that is,

$$
\Delta\left(\mathfrak{g}\left(C_{1}\right), \mathfrak{g}\left(C_{2}\right)\right)=\Delta\left(C_{1}, C_{2}\right)
$$

holds for any Möbius transformation $\mathfrak{g}$. This concept appears in [4], where the term separation is introduced for it, but the concept ${ }^{1}$ was used earlier by Mauldon [25] in 1962, who used the term inclination to mean the negative of $\Delta\left(C_{1}, C_{2}\right)$, and showed it was an inversive invariant. The absolute value of $\Delta\left(C_{1}, C_{2}\right)$ was studied on p. 29 of [2] under the name of an inversive product of two spheres.

The separation $\Delta\left(C_{1}, C_{2}\right)$ of two spheres can be expressed in terms of their augmented curvature-center coordinates as

$$
\begin{aligned}
\Delta\left(C_{1}, C_{2}\right)= & \frac{1}{2} \mathbf{w}\left(C_{1}\right)^{T} \mathbf{K}_{n} \mathbf{w}\left(C_{2}\right) \\
= & -\frac{1}{2}\left(\bar{b}\left(C_{1}\right) b\left(C_{2}\right)\right. \\
& \left.+b\left(C_{1}\right) \bar{b}\left(C_{2}\right)\right)+b\left(C_{1}\right) b\left(C_{2}\right) \sum_{j=1}^{n} x_{j}\left(C_{1}\right) x_{j}\left(C_{2}\right),
\end{aligned}
$$

where $\mathbf{K}_{n}(1)$ is given by

$$
\mathbf{K}_{n}=\left[\begin{array}{rrc}
0 & -1 & 0 \\
-1 & 0 & 0 \\
0 & 0 & 2 I_{n}
\end{array}\right]
$$

This formula can be proved by a simple algebraic calculation. Using it, one can check that for two tangent spheres $C_{1}$ and $C_{2}, \Delta\left(C_{1}, C_{2}\right)=1$, if (1) $C_{1}$ and $C_{2}$ are externally tangent and both are inwardly oriented or outwardly oriented, or (2) $C_{1}$ and $C_{2}$ are internally tangent and one is inwardly oriented, the other is outwardly oriented. In all other cases two tangent spheres have $\Delta\left(C_{1}, C_{2}\right)=-1$, and orthogonal spheres are those with $\Delta\left(C_{1}, C_{2}\right)=0$.

From Theorem 7.1 one obtains

$$
\Delta\left(C^{\perp}, C_{j}\right)=0 \quad \text { for } \quad 1 \leq j \leq n+1,
$$

and these relations determine $C^{\perp}$ up to orientation. It can also be shown that if a set of tangent spheres $\left\{C_{1}, \ldots, C_{n+1}\right\}$ have oriented curvatures $\mathbf{b}_{n+1}=\left(b_{1}, \ldots, b_{n+1}\right)$, and centers $\mathbf{x}_{j}$, then for either orientation the orthogonal sphere $C^{\perp}$ has oriented curvature $q$ satisfying

$$
q^{2}=\frac{1}{2}\left(\frac{1}{n-1}\left(\sum_{j=1}^{n+1} b_{j}\right)^{2}-\sum_{j=1}^{n+1} b_{j}^{2}\right),
$$

\footnotetext{
${ }^{1}$ The idea of considering such an inversive invariant traces back to work by Clifford [8] in 1868 and by Darboux [14] in 1872. However, neither Clifford's nor Darboux' definition was precisely $\Delta\left(C_{1}, C_{2}\right)$. Clifford defines the power of two spheres to be the square distance of their centers less the sum of the squares of their radii, i.e. $d^{2}-r_{1}^{2}-r_{2}^{2}$, and Darboux also uses the same quantity [14, p. 350].
} 
and (oriented) center $\mathbf{x}$ satisfying

$$
q \mathbf{x}=-\mathbf{b}_{n+1}\left(\frac{1}{2} \mathbf{Q}_{D, n-1}\right) \mathbf{C},
$$

in which $\mathbf{C}$ is an $(n+1) \times n$ matrix whose $j$ th row is $b_{j} \mathbf{x}_{j}$.

An oriented Descartes configuration in $\mathbb{R}^{n}$ is characterized in terms of separation as a set of $n+2$ oriented spheres, each pair of which has $\Delta\left(C_{i}, C_{j}\right)=1$, when $i \neq j$. Thus such a configuration has the following property.

Definition 7.2. A collection of oriented spheres is equiseparated if all values $\Delta\left(C_{j}, C_{k}\right)$ with $j \neq k$ are equal.

The equiseparation property can also be viewed as an equiangularity property, because for two oriented circles that intersect or touch one has

$$
\Delta\left(C_{1}, C_{2}\right)=-\cos \theta
$$

where $\theta$ is the angle between oriented normals at a point of intersection of the two circles. We now show the duality operation preserves equiseparability in all dimensions.

Theorem 7.2 (Equiseparation Theorem). Given an oriented Descartes configuration $\mathcal{D}=\left(C_{1}, C_{2}, \ldots, C_{n+2}\right)$ in $\mathbb{R}^{n}$, if the dual spheres are properly oriented then the (oriented) dual configuration $\left(C_{1}^{\perp}, C_{2}^{\perp}, \ldots, C_{n+2}^{\perp}\right)$ is equiseparated, with

$$
\Delta\left(C_{j}^{\perp}, C_{k}^{\perp}\right)=\frac{1}{n-1} \quad \text { if } \quad j \neq k
$$

Proof. In this result the orientation assigned to the dual spheres in the theorem depends on all $n+2$ spheres in the Descartes configuration, and the orientation of $C_{j}^{\perp}$ cannot be consistently assigned from the $n+1$ oriented spheres $\left\{C_{i}: i \neq j\right\}$ alone. If all $n+2$ spheres $C_{j}$ are inwardly oriented, then $n+1$ of the spheres $C_{j}^{\perp}$ will be inwardly oriented and one outwardly oriented, the last being the one of largest radius. If all but one of the $n+2$ spheres are inwardly oriented, and one outwardly oriented, then all $n+2$ spheres $C_{j}^{\perp}$ will be inwardly oriented.

Since the result is invariant under inversion, it suffices to prove it for a single Descartes configuration. We consider the special oriented Descartes configuration where the curvatures are $(0,0,1,1, \ldots, 1)$. Here we have two parallel planes, which we take as $x_{1}= \pm 1$, and $n$ unit spheres, all with centers on the plane $x_{1}=0$. Their centers form a regular simplex in this plane. We may take one of these centers at $(0, \xi, 0,0, \ldots)$ where $\xi^{2}=2(n-1) / n$. Consider the "orthogonal" spheres that pass through the point $T=(1, \xi, 0,0, \ldots, 0)$. There are $n$ such spheres, and all but one of them is a hyperplane containing the points $T$ and $(-1, \xi, 0,0 \ldots, 0)$, and the centers of all but one of the original unit spheres. These centers form the vertices of a regular $(n-1)$-simplex, so these $n-1$ "orthogonal" planes are equiangular satisfying (7.12), where $\theta$ is the angle between the normals of two facets of a regular $n$-simplex. It follows that these orthogonal planes satisfy (7.13). The final "orthogonal" sphere through $T$ is orthogonal to the plane $x_{1}=1$ and all the $n$ original unit spheres. Its center is thus $(1,0,0, \ldots)$ 
and its radius is $\xi$. Hence it is also equiangular with the $n-1$ "orthogonal" planes, with $\cos \theta_{n}=-1 /(n-1)$. These angles are all equal to the one formed by connecting the vertices of a regular simplex to its center, i.e. the angle in a triangle of sides $\xi, \xi$ and 2. Finally, the last two "orthogonal" spheres meet at the same angle in the plane $x_{1}=0$.

\section{Concluding Remarks}

This paper studied generalizations of the basic properties of two-dimensional Apollonian packings and super-Apollonian packings. We gave fairly complete answers but left open a few problems.

The first problem is to determine a presentation of the super-Apollonian group $\mathcal{A}_{n}^{S}$ in dimensions $n \geq 3$, in terms of the given generators. Is this group always a hyperbolic Coxeter group?

There are some unanswered questions concerning rational and integral structures on Descartes configurations. In Section 3.1 we raised the question of determining those dimensions $n$ in which the Descartes form $Q_{D, n}$, the Wilker form $Q_{W, n}$ and the Lorentz form $Q_{\mathcal{L}, n}$ are rationally equivalent. In Section 6 we gave a necessary and sufficient condition for rational equivalence of the pair (Descartes, Wilker), namely, that $n=2 k^{2}$ or $n=(2 k-1)^{2}$. For the other two pairs, (Descartes, Lorentz) and (Wilker, Lorentz), we gave the necessary conditions $n=2 k^{2}$ and $n=2 k$, respectively. It remains to determine necessary and sufficient conditions in these cases.

Finally, we left open the question of whether there is any dimension $n \geq 3$ in which there exist strongly integral Descartes configurations. Although strong integrality will not be preserved under the action of the super-Apollonian group (since $n \geq 4$ ), it would be preserved under the action of the dual Apollonian group $\mathcal{A}_{n}^{\perp}$, which consists of integer matrices in all dimensions.

\section{Acknowledgments}

The authors are grateful for helpful comments from Andrew Odlyzko and N. J. A. Sloane. The authors thank the reviewer for incisive comments leading to reorganization of the paper.

\section{Appendix. Möbius Group Action}

The Möbius group action given in Appendix A of Part I straightforwardly extends to $n$-dimensions.

The (general) Möbius group $\operatorname{Möb}(n)$ is the group generated by reflections in spheres or planes in the one-point compactification $\hat{\mathbb{R}}^{n}=\mathbb{R}^{n} \cup\{\infty\}$ of $\mathbb{R}^{n}$, see Chapter 3 of [2]. (Beardon denotes it $G M\left(\hat{\mathbb{R}}_{n}\right)$.) This group has two connected components, and we let $\operatorname{Möb}(n)_{+}$denote the connected component of the identity. The extended Möbius group $G M^{*}(n)$ is defined by $G M^{*}(n):=\operatorname{Möb}(n) \times\{-\mathbf{I}, \mathbf{I}\}$. Here $\{-\mathbf{I}, \mathbf{I}\}$ are in the center of 
Table 1. Group isomorphisms.

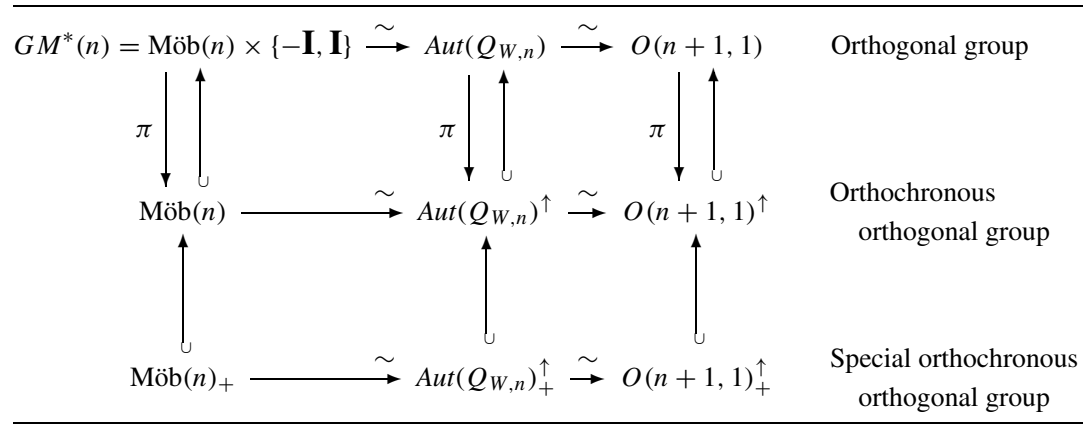

this group, and we write elements of $G M^{*}(n)$ as $\pm \mathfrak{g}$, in which $\mathfrak{g} \in \operatorname{Möb}(n)$, and the sign indicates which of $\pm \mathbf{I}$ occurs. The group $G M^{*}(n)$ has four connected components.

The purpose of this Appendix is to define an action of $G M^{*}(n)$ on the right on the parameter space $\mathcal{M}_{\mathbb{D}}^{n}$, given in Theorem A.1 below. This amounts to finding an explicit isomorphism between $G M^{*}(n)$ and $\operatorname{Aut}\left(Q_{W, n}\right)$. The case $n=2$ was treated in Appendix A of Part I [18, Theorem 7.2], where it was shown that the Möb(2) action preserves (total) orientation of Descartes configurations. The same property holds for the Möb $(n)$ action treated here, by a similar proof which we omit.

Relevant isomorphisms are given in Table 1. The isomorphism between $G M^{*}(n)$ and $\operatorname{Aut}\left(Q_{W, n}\right)$ appears as the horizontal arrow on the left in the top row. This map when restricted to the smaller groups $\operatorname{Möb}(n)$ and $\operatorname{Möb}(n)_{+}$, gives the other two horizontal isomorphisms on the left side of the table. Table 1 also indicates isomorphisms on its right side to the orthogonal group $O(n+1,1)$ and corresponding subgroups, which we defer discussing until after the following result.

Theorem A.1. Let $G M^{*}(n):=\operatorname{Möb}(n) \times\{\mathbf{I},-\mathbf{I}\}$. There is a unique isomorphism $\pi: G M^{*}(n) \rightarrow \operatorname{Aut}\left(Q_{W, n}\right)$, with image elements $\mathbf{V}_{ \pm \mathfrak{g}}:=\pi( \pm \mathfrak{g})$, such that the following hold:

(i) For $\mathfrak{g} \in \operatorname{Möb}(n)$ the augmented curvature-center coordinates for each ordered, oriented Descartes configuration D satisfy

$$
\mathbf{W}_{\mathfrak{g}(\mathcal{D})}=\mathbf{W}_{\mathcal{D}} \mathbf{V}_{\mathfrak{g}}^{-1} .
$$

(ii) The action of $\mathbf{- I}$ on augmented curvature-center coordinates is

$$
\mathbf{W}_{-\mathcal{D}}=\mathbf{W}_{\mathcal{D}} \mathbf{V}_{-\mathbf{I}}^{-1}=-\mathbf{W}_{\mathcal{D}} .
$$

This reverses the orientation of the Descartes configuration.

Proof. We compute the action of $\operatorname{Möb}(n)$ on augmented curvature-center coordinates. Let $\left(\bar{b}, b, w_{1}, w_{2}, \ldots, w_{n}\right)=\left(\left(\left(\sum_{i=1}^{n} x_{i}^{2}\right)-r^{2}\right) / r, 1 / r, x_{1} / r, x_{2} / r, \ldots, x_{n} / r\right)$ be the augmented curvature-center coordinates of the sphere

$$
\sum_{i=1}^{n}\left(y_{i}-x_{i}\right)^{2}=r^{2} .
$$


This sphere can be recovered from these coordinates via

$$
\sum_{i=1}^{n}\left(b y_{i}-w_{i}\right)^{2}=1
$$

and the orientation of the sphere (inside versus outside) is determined by the sign of $b$. An oriented "sphere at infinity" is a hyperplane given by

$$
\mathbf{y} \cdot \mathbf{h}=m,
$$

and its associated curvature-center coordinates are

$$
\left(\bar{b}, b, w_{1}, w_{2}, \ldots, w_{n}\right)=\left(2 m, 0, h_{1}, h_{2}, \ldots, h_{n}\right),
$$

where $\mathbf{h}=\left(h_{1}, h_{2}, \ldots, h_{n}\right)$ is the unit normal vector, and the orientation is given by the convention that the normal $\mathbf{h}$ points inward.

The group $\operatorname{Möb}(n)$ is generated by

(1) translations $\mathbf{t}_{\mathbf{y}_{0}}(\mathbf{y})=\mathbf{y}+\mathbf{y}_{0}$;

(2) dilations $\mathfrak{d}_{r}(\mathbf{y})=r \mathbf{y}$ with $r \in \mathbb{R}, r>0$;

(3) the rotation $\mathfrak{o}(\mathbf{y})=\mathbf{O y}$, where $\mathbf{O}$ is an $n \times n$ orthogonal matrix;

(4) the inversion in the unit circle $\mathfrak{j}_{C}(\mathbf{y})=\mathbf{y} /|\mathbf{y}|^{2}$.

Given $\mathfrak{g} \in \operatorname{Möb}(n)$, we let $\tilde{\mathfrak{g}}$ denote the corresponding action on the curvature-center coordinates of an oriented circle. The action of translation by $\mathbf{y}_{0}=\left(y_{0,1}, y_{0,2}, \ldots, y_{0, n}\right)$ is

$$
\begin{aligned}
\tilde{\mathfrak{t}}_{\mathbf{y}_{0}}\left(\bar{b}, b, w_{1}, w_{2}, \ldots, w_{n}\right) \\
=\left(\bar{b}+2 \sum_{i=1}^{n} w_{i} y_{0, i}+b \sum_{i=1}^{n} y_{0, i}^{2}, b, w_{1}+b y_{0,1}, w_{2}+b y_{0,2}, \ldots, w_{n}+b y_{0, n}\right) .
\end{aligned}
$$

The action of a dilation with $r \in \mathbb{R}(r>0)$ is given by

$$
\tilde{\mathfrak{d}}_{\lambda}\left(\bar{b}, b, w_{1}, w_{2}, \ldots, w_{n}\right)=\left(r \bar{b}, b / r, w_{1}, w_{2}, \ldots, w_{n}\right) .
$$

The action of rotation $\mathfrak{o}$ with orthogonal matrix $\mathbf{O}$ is

$$
\tilde{\mathfrak{o}}\left(\bar{b}, b, w_{1}, w_{2}, \ldots, w_{n}\right)=\left(\bar{b}, b, w_{1}^{\prime}, w_{2}^{\prime}, \ldots, w_{n}^{\prime}\right),
$$

where $\left(w_{1}^{\prime}, w_{2}^{\prime}, \ldots, w_{n}^{\prime}\right)=\left(w_{1}, w_{2}, \ldots, w_{n}\right) \mathbf{O}^{T}$. The action of inversion in the unit circle is

$$
\tilde{\mathfrak{j}}_{C}\left(\bar{b}, b, w_{1}, w_{2}, \ldots, w_{n}\right)=\left(b, \bar{b}, w_{1}, w_{2}, \ldots, w_{n}\right) .
$$

All of these actions apply to "spheres at infinity" and extend to linear maps on the $(n+2) \times(n+2)$ matrices $\mathbf{W}_{\mathcal{D}}$.

The translation operation is given by right multiplication by the matrix

$$
\mathbf{V}_{\mathbf{t y}_{0}}^{-1}:=\left[\begin{array}{ccccc}
1 & 0 & 0 & \ldots & 0 \\
\sum_{i=1}^{n} y_{0, i}^{2} & 1 & y_{0,1} & \ldots & y_{0, n} \\
2 y_{0,1} & 0 & \ddots & & \\
\vdots & \vdots & & \mathbf{I}_{n} & \\
2 y_{0, n} & 0 & & & \ddots
\end{array}\right] \text {, }
$$


and one verifies (A.1) holds by direct computation. For the dilation $\mathfrak{d}_{r}$, with $r \in \mathbb{R}, r>0$ the right action is by the matrix

$$
\mathbf{V}_{\mathfrak{d}_{r}}^{-1}:=\left[\begin{array}{ccc}
r & 0 & 0 \\
0 & 1 / r & 0 \\
0 & 0 & \mathbf{I}_{n}
\end{array}\right] .
$$

For rotation $\mathfrak{o}$, the right action is by the matrix

$$
\mathbf{V}_{\mathfrak{o}}^{-1}=:=\left[\begin{array}{ccc}
1 & 0 & 0 \\
0 & 1 & 0 \\
0 & 0 & \mathbf{O}^{T}
\end{array}\right] .
$$

For the inversion $\mathfrak{j}_{C}$ in the unit circle, the permutation matrix

$$
\mathbf{V}_{\mathrm{j}_{c}}^{-1}=\mathbf{V}_{\mathrm{j}_{c}}=\mathbf{P}_{(12)}=\left[\begin{array}{ccc}
0 & 1 & 0 \\
1 & 0 & 0 \\
0 & 0 & \mathbf{I}_{n}
\end{array}\right] .
$$

It is easy to verify that the above matrices are all in $\operatorname{Aut}\left(Q_{W}\right)$. Since $\mathbf{Q}_{W, n}=\mathbf{A}^{T} \mathbf{Q}_{\mathcal{L}, n} \mathbf{A}$ where

$$
\mathbf{A}=\sqrt{2}\left[\begin{array}{rrr}
1 & 1 & 0 \\
-1 & 1 & 0 \\
0 & 0 & \mathbf{I}_{n}
\end{array}\right]
$$

we have that a matrix $\mathbf{U} \in \operatorname{Aut}\left(Q_{W, n}\right)^{\uparrow}$ if and only if $\mathbf{e}_{1}^{T} \mathbf{A} \mathbf{U} \mathbf{A}^{-1} \mathbf{e}_{1}>0$. One checks that all the above matrices are actually in $\operatorname{Aut}\left(Q_{W, n}\right)^{\uparrow}$, so that the map so far defines a homomorphism of $\operatorname{Möb}(n)$ into $\operatorname{Aut}\left(Q_{W, n}\right)^{\uparrow} \simeq O(n+1,1)^{\uparrow}$, identified with the isochronous Lorentz group. The group $\operatorname{Möb}(n)$ acts simply transitively on ordered Descartes configurations, as observed by Wilker [35, Theorem 3, p. 394], and the group Aut $\left(Q_{W, n}\right)$ acts simply transitively on ordered, oriented Descartes configurations, as implied by Theorem 3.1. Because $\operatorname{Aut}\left(Q_{W, n}\right)^{\uparrow}$ is of index 2 in $\operatorname{Aut}\left(Q_{W, n}\right) \simeq O(n+1,1)$, we conclude that the map so far defines an isomorphism of $\operatorname{Möb}(n)$ onto $\operatorname{Aut}\left(Q_{W, n}\right)^{\uparrow}$.

To complete the proof, we define the action of $-I$ to be

$$
\left(\mathbf{V}_{-I}\right)^{-1}=\mathbf{V}_{-I}=-\mathbf{I}_{n+2} .
$$

It has the effect of reversing (total) orientation of the Descartes configuration, and does not correspond to a conformal transformation. Since $-\mathbf{I}_{n+2} \notin \operatorname{Aut}\left(Q_{W, n}\right)^{\uparrow}$, adding it gives the desired isomorphism of $G M^{*}(n)$ onto $\operatorname{Aut}\left(Q_{W, n}\right)$.

We now return to the data in Table 1, giving the isomorphisms of $\operatorname{Aut}\left(Q_{W, n}\right)$ and its subgroups to the orthogonal group $O(n+1,1)$ and its two subgroups $O(n+1,1)^{\uparrow}$, the orthochronous orthogonal group, and $O(n+1,1)_{+}^{\uparrow}$, the special orthochronous orthogonal group, which is the connected component of the identity of the orthogonal group $O(n+$ $1,1)$. The set of isomorphisms given by the three horizontal arrows on the right in Table 1 are obtained by any fixed choice of real matrix $\mathbf{A}$ that intertwines $Q_{W, n}$ and $Q_{\mathcal{L}, n}$ by $\mathbf{Q}_{W}=\mathbf{A}^{T} \mathbf{Q}_{\mathcal{L}, n} \mathbf{A}$, in which case the isomorphism is $\operatorname{Aut}\left(Q_{W, n}\right)=\mathbf{A}^{-1} O(n+1,1) \mathbf{A}$ sending $\mathbf{V} \mapsto \mathbf{A V A}^{-1}$. Such matrices $\mathbf{A}$ exist in all dimensions. It is shown in Section 3.1 that rational matrices $\mathbf{A}$ do not exist in all dimensions; a necessary condition for existence is that the dimension $n$ be even. 


\section{Bibliography}

1. D. Aharonov and K. Stephenson, Geometric sequences of discs in the Apollonian packing, Algebra $i$ Analiz 9(3) (1997), 104-140. (English version: St. Petersburg Math. J. 9 (1998), 509-545.)

2. A. F. Beardon, The Geometry of Discrete Groups, Springer-Verlag, New York, 1983.

3. M. Berger, Geometry II, Springer-Verlag, Berlin, 1987.

4. D. W. Boyd, The osculatory packing of a three-dimensional sphere, Canad. J. Math. 25 (1973), 303-322.

5. D. W. Boyd, A new class of infinite sphere packings, Pacific J. Math. 50 (1974), 383-398.

6. R. W. Brooks, The spectral geometry of the Apollonian packing, Comm. Pure. Appl. Math. 38 (1985), 359-366.

7. J. W. S. Cassels, Rational Quadratic Forms, Academic Press, New York, 1978.

8. W. K. Clifford, On the powers of spheres (1868), in: Mathematical Papers of William Kingdon Clifford, MacMillan, London, 1882, pp. 332-336.

9. J. H. Conway, with F. Fung, The Sensual (Quadratic) Form, Carus Monograph No. 26, Mathematical Association of America, Washington, DC, 1997.

10. H. S. M. Coxeter, The problem of Apollonius, Amer. Math. Monthly 75 (1968), 5-15.

11. H. S. M. Coxeter, Loxodromic sequences of tangent spheres, Aequationes Math. 1 (1968), 104-121.

12. H. S. M. Coxeter, Introduction to Geometry, second edition, Wiley, New York, 1969.

13. H. S. M. Coxeter, Numerical distances among the spheres in a loxodromic sequence, Math. Intelligencer 19 (1997), 41-47.

14. G. Darboux, Sur les relations entre les groupes de points, de cercles et de sphéres dans le plan et dans l'espace, Ann. Sci. École Norm. Sup. 1 (1872), 323-392.

15. K. J. Falconer, The Geometry of Fractal Sets, Cambridge Tracts in Mathematics, vol. 85, Cambridge University Press, Cambridge, 1986.

16. T. Gossett, The Kiss Precise, Nature 139 (1937), 62.

17. T. Gossett, The Hexlet, Nature 139 (1937), 251.

18. R. L. Graham, J. C. Lagarias, C. L. Mallows, A. Wilks and C. Yan, Apollonian circle packings: geometry and group theory. I. Apollonian group, Discrete Comput. Geom. DOI: 10.1007/s00454-005-1196-9. [eprint: arXiv math.MG/0010298]

19. R. L. Graham, J. C. Lagarias, C. L. Mallows, A. Wilks and C. Yan, Apollonian circle packings: geometry and group theory. II. Super-Apollonian group and integral packings, Discrete Comput. Geom. DOI: 10.1007/s00454-005-1195-x. [eprint: arXiv math.MG/0010302]

20. R. L. Graham, J. C. Lagarias, C. L. Mallows, A. Wilks and C. Yan, Apollonian circle packings: number theory, J. Number Theory 100 (2003), 1-45. [eprint: arXiv math. NT/009113]

21. E. Kasner and F. Supnick, The Apollonian packing of circles, Proc. Nat. Acad. Sci. USA 29 (1943), 378-384.

22. R. Lachlan, On systems of circles and spheres, Philos. Trans. Roy. Soc. London Ser. A 177 (1886), 481-625.

23. J. C. Lagarias, C. L. Mallows and A. Wilks, Beyond the Descartes circle theorem, Amer. Math. Monthly 109 (2002), 338-361. [eprint: arXiv math.MG/0101066]

24. B. B. Mandelbrot, The Fractal Geometry of Nature, Freeman, New York, 1983.

25. J. G. Mauldon, Sets of equally inclined spheres, Canad. J. Math. 14 (1962), 509-516.

26. G. Maxwell, Sphere packings and hyperbolic reflection groups, J. Algebra 79 (1982), 78-97.

27. Z. A. Melzak, Infinite packings of disks, Canad. J. Math. 18 (1966), 838-853.

28. D. Pedoe, On a theorem in geometry, Amer. Math. Monthly 74 (1967), 627-640.

29. B. Rodin and D. Sullivan, The convergence of circle packings to the Riemann mapping, J. Differential Geom. 26 (1987), 349-360.

30. F. Soddy, The Kiss Precise, Nature 137 (1936), 1021.

31. F. Soddy, The Hexlet, Nature 138 (1936), 958.

32. F. Soddy, The Bowl of Integers and the Hexlet, Nature 139 (1937), 77-79.

33. F. Soddy, The Hexlet, Nature 139 (1937), 154.

34. B. Söderberg, Apollonian tiling, the Lorentz group, and regular trees, Phys. Rev. A 46(4) (1992), 18591866.

35. J. B. Wilker, Inversive geometry, in: The Geometric Vein (C. Davis, B. Grünbaum and F. A. Sherk, Eds.), Springer-Verlag, New York, 1981, pp. 379-442.

Received October 31, 2000, and in revised form March 11, 2005. Online publication October 17, 2005. 